\title{
XMM-Newton observation of M 87
}

\section{Single-phase temperature structure of intracluster medium}

\author{
K. Matsushita ${ }^{1}$, E. Belsole ${ }^{2}$, A. Finoguenov ${ }^{1}$, and H. Böhringer ${ }^{1}$ \\ 1 Max-Planck-Institut für Extraterrestrial Physik, 85748 Garching, Germany \\ 2 Service d'Astrophysique, CEA Saclay, L'Orme des Merisiers, Bât. 709, 91191 Gif-sur-Yvette Cedex, France
}

Received 19 June 2001 / Accepted 9 January 2002

\begin{abstract}
We report the results of a detailed analysis of the temperature structure of the X-ray emitting plasma halo of M 87, the cD galaxy of the Virgo Cluster. Using the MEKAL model, the data provide strong indications that the intracluster medium has a single phase structure locally, except the regions associated with the radio structures. The deprojected spectrum at each radius is well fitted by a single temperature MEKAL model, except for the very central region $(<2$ arcmin) which seems to be affected by the jet and radio lobe structure. The temperature of the intracluster plasma is $1 \mathrm{keV}$ at the center and gradually increases to $2.5 \mathrm{keV}$ at $80 \mathrm{kpc}$. We have also fitted spectra using the APEC code. Although the large changes of the strength of K $\alpha$ lines causes a discrepancy between the Fe-L and Fe-K lines for the APEC results, the overall temperature structure has not changed. There is no sign of excess absorption in the spectral data. The single-phase nature of the intracluster medium is in conflict with the standard cooling flow model which is based on a multi-phase temperature structure. In addition, the signature of gas cooling below $0.8 \mathrm{keV}$ to zero temperature is not observed as expected for a cooling flow. The gravitational mass profile derived from the temperature and density distribution of the intracluster gas shows two distinct contributions that can be assigned to the gravitational potential of the cD galaxy and the cluster. The central temperature of the intracluster medium agrees well with the potential depth and the velocity dispersion of the $\mathrm{cD}$ galaxy. The latter result implies that the central region of the intracluster medium is equivalent to a virialized interstellar medium in $\mathrm{M} 87$.
\end{abstract}

Key words. X-rays: galaxies - galaxies: ISM - galaxies: individual: M 87

\section{Introduction}

In the cores of many clusters of galaxies, X-ray imaging data show a highly peaked surface brightness profile (e.g. Fabian et al. 1981). The radiative cooling time in these regions is much less than a Hubble time. Without a heating process, the gas cools to low temperature and results in a "cooling flow" (Fabian 1994 for a review). The mass flow rate, $\dot{M}$, that is deduced in the standard cooling flow model, is approximately proportional to the radius. This implies that matter is deposited throughout the entire cooling flow region. It also implies that the gas in the cooling flow zone is "multi-phase" on scales small enough that the inhomogeneities have escaped the observation so far.

ASCA and ROSAT observations confirmed the existence of cooler gas in the cores of cooling flow clusters as expected (e.g. Allen \& Fabian 1994; Ikebe et al. 1999; Ikebe 2001). The presence of an intrinsic absorber in excess of the galactic absorption, as it might be expected

Send offprint requests to: K. Matsushita,

e-mail: matusita@xray.mpe.mpg.de to arise from the accumulation of cold gas in the cooling flows, was also inferred from the analysis of the ASCA spectra (e.g. Allen 2000; Allen et al. 2001). In fact, the fitting of the "multi-phase" cooling flow model to the observed spectra including temperature phases that cool below the X-ray emitting temperature regime, requires the inclusion of excess absorption to satisfactorily reproduce the observed spectra. It has also been argued, that inclusion of excess absorption leads to values of the mass flow rate as obtained from imaging data, $\dot{M}_{\mathrm{I}}$ in good agreement with those obtained from spectral analysis, $\dot{M}_{\mathrm{S}}$ (Allen 2000).

However, without assuming excess absorption, the values of $\dot{M}_{\mathrm{S}}$ are systematically lower than $\dot{M}_{\mathrm{I}}$ (e.g. Ikebe et al. 1999; Makishima et al. 2001). Instead of the cooling flow model, a two temperature spectral model can well fit ASCA spectra of the cooling flow clusters. The temperatures of the softer component are about half of the hotter component (Ikebe 2001). The central temperatures of some $\mathrm{cD}$ galaxies obtained with the ROSAT PSPC are close to those obtained from normal elliptical galaxies with 
the same stellar velocity dispersion (Matsushita 2001). Since cooling flow clusters always possess central dominant galaxies, the cool components may reflect the potential structure, rather than cooling gas (Ikebe et al. 1999; Makishima et al. 2001; Ikebe 2001).

Recently, it was discovered with the RGS instrument onboard XMM-Newton that there is little X-ray emission from a component with a temperature below a certain lower cutoff value that differs from object to object (e.g. Tamura et al. 2001; Kaastra et al. 2001). However, with the RGS one observes only the very central part of the cluster.

M 87 is the $\mathrm{cD}$ galaxy of the nearest rich cluster of galaxies, the Virgo Cluster. It is very luminous in X-rays, and is suggested to have a "cooling flow" with $\dot{M}$ of about $10 M_{\odot} \mathrm{yr}^{-1}$ (Stewart et al. 1984; Fabian et al. 1984). M 87 also hosts a central active galactic nuclei (AGN). The radio emission is complex and there are two strong lobe structures (e.g. Böhringer et al. 1995). An enhancement of the X-ray emission around the lobes was discovered with the EINSTEIN HRI (Feigelson et al. 1987) and ROSAT PSPC (Böhringer et al. 1995).

In addition to the XMM RGS results mentioned above, the XMM-Newton observatory offers the possibility to perform a very detailed spectral and spatial analysis of the cooling flow area. This is in particular important since the RGS spectrum taken at the very central part of the intracluster medium (ICM) may be strongly affected by resonance line scattering and thus the interpretation of the results requires the combined analysis of spectra taken at different radii from the center. Early results of the XMM observation of M 87 have already been published (Böhringer et al. 2001; Belsole et al. 2001). The projected spectrum is fitted with a single temperature model (Böhringer et al. 2001). The abundance drop at the central region is interpreted as resonance scattering. The detailed spectral analysis on the jets and the radio lobe structure is shown in Belsole et al. (2001).

In this paper, we report on the more detailed temperature structure using a deprojecting analysis, and apply a cooling flow model to the spectral analysis. We employ a distance of M 87 of $17 \mathrm{Mpc}$. We adopt for the solar iron abundance the "photospheric" value, $\mathrm{Fe} / \mathrm{H}=4.68 \times 10^{-5}$ by number (Anders \& Grevesse 1989). Unless otherwise specified, we use $90 \%$ confidence error regions.

\section{Observation and data preparation}

M 87 was observed with XMM-Newton on June 19th, 2000. The thin filter is used for both detectors.

\section{1. $E P N$}

Figure 1 shows the X-ray image of M 87 obtained with the EPN. The contribution from the out-of-time events is subtracted following the standard analysis package (SAS). The image divided by an azimuthally symmetric image with the same radial profile is overlayed in Fig. 1.

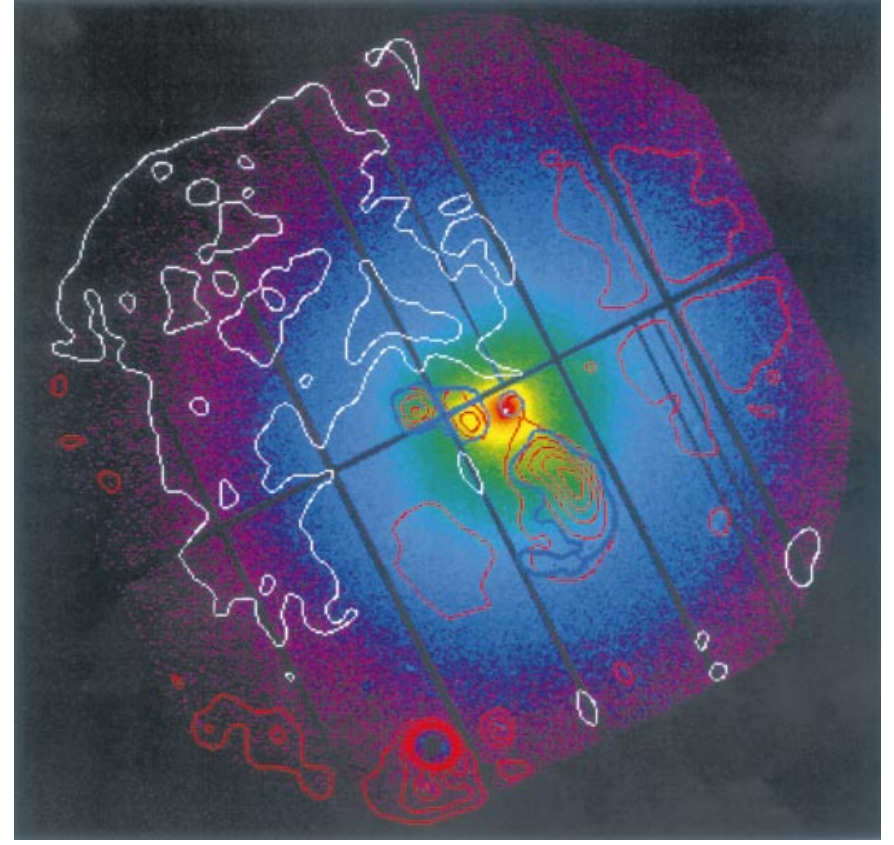

Fig. 1. EPN image of M 87 in the energy range of 0.2 to $10.0 \mathrm{keV}$. The effect of the out-of-time events is corrected. The overlayed contours correspond to the image divided by a azimuthally symmetric with the same radial profile, smoothed with $20^{\prime \prime}$ Gaussian. The contour levels are 0.8, 0.9 (white), 1.1, $1.2,1.3,1.4$, and 1.5 (red). The regions surrounded by the blue contours are excluded from our spectral analysis as described in Sect. 3.3.

The X-ray halo is very smooth and almost spherically symmetric within $20 \%$, except for the two inner radio lobe regions and the first knot in the jet. A luminous point source near the south edge of the detector is excised in the further analysis.

We have selected single pattern events. The background spectrum was calculated for all spectra by integrating the Lockman-Hole data (observed in the Revolution 70, 71, 73, 81 for the EPN) in the same detector regions. In the Lockman-Hole field, sources with a luminosity higher than that of the excluded sources from the $\mathrm{M} 87$ region are excised. The flare events in the background data were screened in the following way. We made two count rate histograms of bin width of $200 \mathrm{~s}$, for the corner region in the whole energy band, and for the whole detector for above $10 \mathrm{keV}$. Then, we fitted each histogram with a Gaussian, and selected the time within $3 \sigma$ of the mean for both histograms. The total exposure of the background data is $95 \mathrm{ks}$. We have also screened the M 87 data in the same way. The exposure times of the EPN are $30 \mathrm{ks}$, $28 \mathrm{ks}, 27 \mathrm{ks}, 30 \mathrm{ks}$ for the Chips 1-3, 4-6, 7-9, and 10-12, respectively.

The spectra of each CCD chip are accumulated within a ring, centered on M 87. Then, we have subtracted the background and corrected the effect of the vignetting and exposure, and summed the spectra within annular regions. The contribution of out-of-time events is subtracted using SAS. 


\subsection{EMOS}

We have selected events with pattern between 0 to 12 . The background spectra are accumulated from the LockmanHole data observed in Revolution 70. The background flare events are screened in the same way for the EPN. The exposure times of the background and data are $33 \mathrm{ks}$ and $40 \mathrm{ks}$, respectively. Out-of-time events are not subtracted since the contribution is negligible.

\subsection{Spectral deprojection}

Deprojected spectra are calculated by subtracting the contribution from the outer shell regions for all spectral components, assuming the ICM is spherically symmetric as done by Nulsen \& Böhringer (1995). Within a each shell, the spectrum per unit volume is assumed to be the same. In order to calculate the deprojected spectrum of the outermost ( $N$ th) shell, we subtracted the emission of the outside of the $N$ th shell using the brightness profile derived from the ROSAT All Sky Survey (Böhringer et al. 1994) assuming that spectral shape outside the $N$ th shell is the same with that of the $N$ th shell. The deprojected spectrum of the $i$ th shell is then calculated by subtracting the contributions from $i+1$ th to $N$ th shell and from outside of the $N$ th shell from the annular spectrum of the corresponding radius.

\section{Spatial distribution of temperature components}

\subsection{Radial temperature profile}

We employ epn_fs20_sY9_thin.rmf from March 2001, m1_thin1v9q19t5r5_all_15.rsp from June 2001 for the EPN and the EMOS response files, respectively. The spectral analysis uses the XSPEC_v11.0.1 package.

Figure 2 shows annular spectra for the radial range $r=5.6-8^{\prime}$ of the EPN and the EMOS, fitted with a same single temperature MEKAL model (Mewe \& Gronenschild 1985; Mewe et al. 1986; Kaastra 1992; Liedahl et al. 1995) but different normalization. Here, $r$ is the the projected radius. The EMOS gives about $10 \%$ higher normalizations than the EPN. The response matrices of the EPN and EMOS are mostly consistent. Below $0.5 \mathrm{keV}$, EPN data is lower than the EMOS data, and there are small discrepancies around the tail of the Fe-L lines at $0.8-1.0 \mathrm{keV}$ and around the instrumental Si edge at $2 \mathrm{keV}$. Since the EMOS gives a hydrogen column density, $N_{\mathrm{H}}$, consistent with that of ROSAT (cf. Sect. 6), we fitted to the EPN and EMOS data in the spectral range of 0.5 to $10 \mathrm{keV}$ and 0.3 to $10 \mathrm{keV}$, respectively. For $R>8^{\prime}$, the energy range between 7.5 and $8.5 \mathrm{keV}$ of the EPN spectra is ignored, because of strong instrumental emission lines.

We have fitted the spectra for the region with radius $R>0.5^{\prime}$ with a MEKAL model with photoelectric absorption. Here, $R$ is the three dimensional radius. For the spectra within $0.5^{\prime}$, a power-law component, with the same absorption, $N_{\mathrm{H}}$, is added. When fitting the spectra between

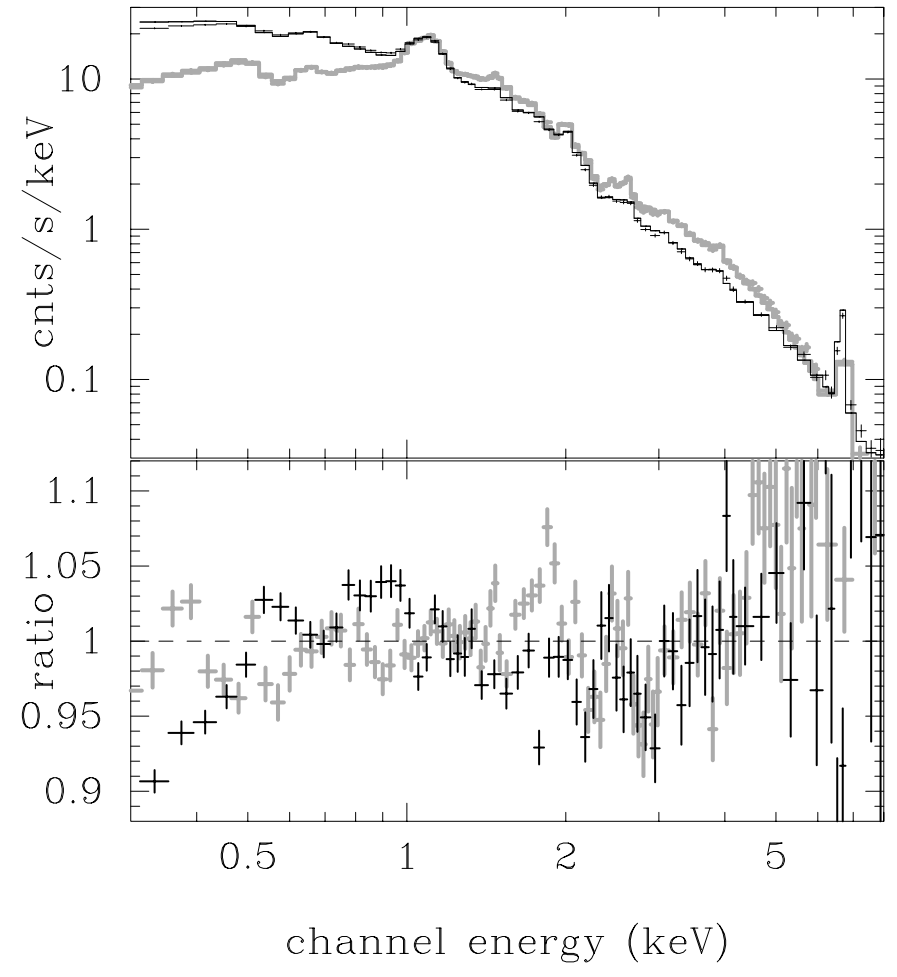

Fig. 2. Annular spectra of the EPN (black) and EMOS (EMOS1+EMOS2; gray) for $r=5.6-8^{\prime}$, fitted with a same single temperature MEKAL model. Bottom panels show datato-model ratios.

$0.12^{\prime}$ and $0.5^{\prime}$, we fixed the index of the power-law component to the best-fit value obtained from the spectrum within $0.12^{\prime}$ and normalized it using the Point Spread Function. We grouped several elements and constrained the elements in each group to have a common abundance. The first group is $\mathrm{C}, \mathrm{N}$, and $\mathrm{O}$, and the second group is Fe and $\mathrm{Ni}$. The other elements are determined separately. We also fitted the spectra with a sum of two MEKAL models, where the two components were constrained to have common abundances.

We have summarized the results in Table 1.

Figures 3 and 4 show the radial profile of the temperature obtained by the single temperature model, and the two temperature model, respectively. The temperatures derived from the EPN and EMOS are mostly consistent.

Within $R<6^{\prime}$ and $R<13.5^{\prime}$ of the EMOS and EPN respectively, the two temperature model gives significantly lower $\chi^{2}$ values than the single temperature model. The temperature of the cooler component is almost constant at $\sim 1 \mathrm{keV}$, which is the same as the central temperature obtained by the single temperature model fit. That of the hotter component show a nearly constant value at $R<2^{\prime}$, whereas for $R>2^{\prime}$ it increases gradually. In Fig. 5, we have plotted the ratio of the emission measure of the cool to the hot component, assuming the temperature of the former to be $1.0 \mathrm{keV}$. The fraction of the cool component is $20 \%$ at $R=0.5^{\prime}$ and drops below $1 \%$ beyond $6^{\prime}$ for the EMOS. Beyond $4^{\prime}$, the EPN tends to have $1-2 \%$ larger values of the contribution of the cooler component than 
Table 1. Result of spectrum fitting of the deprojected spectra (whole region).

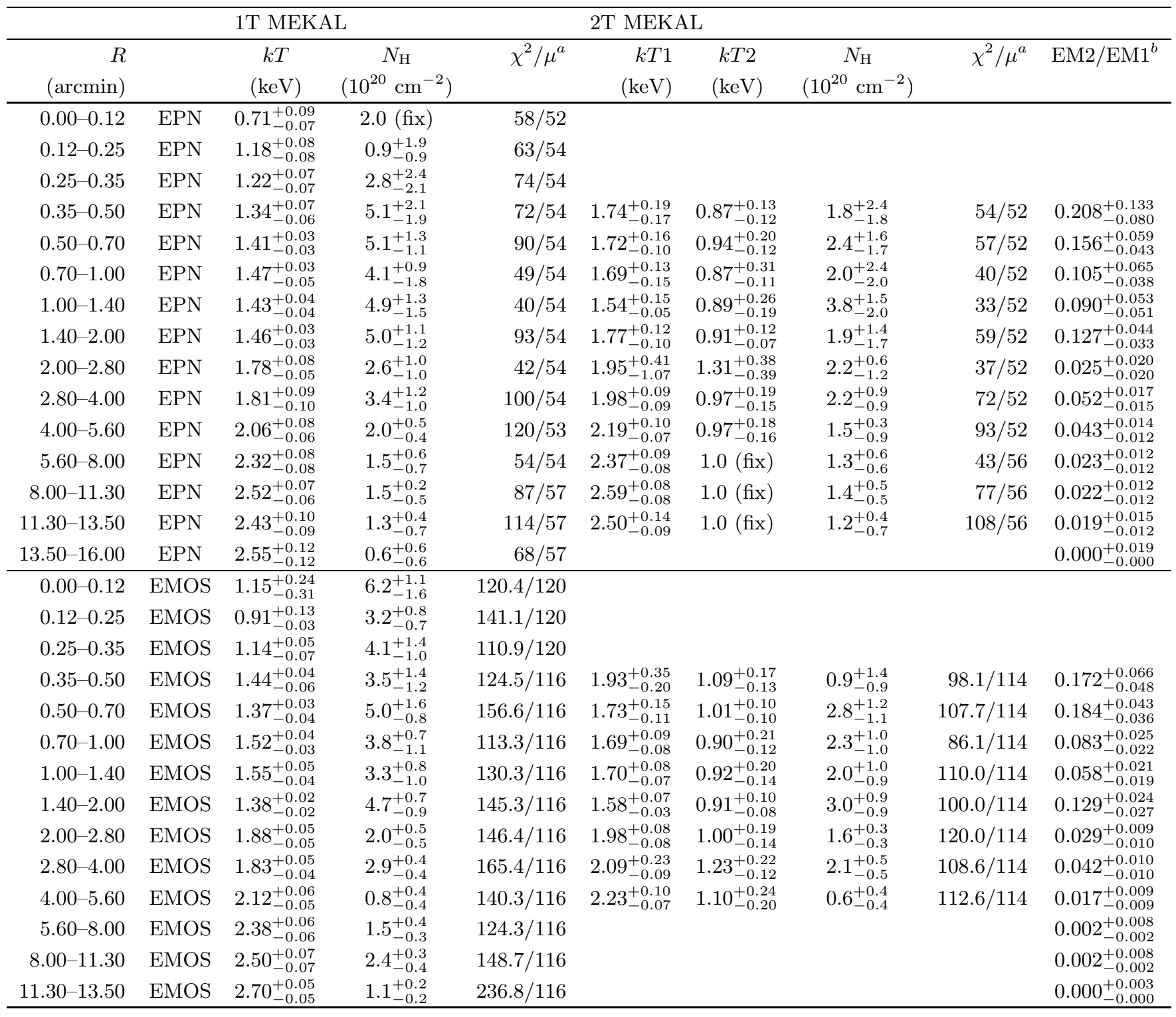

${ }^{a}$ Degrees of freedom.

${ }^{b}$ Ratio of emission measure of the cooler and hotter component, when the temperature of the former is fixed to $1 \mathrm{keV}$.

the EMOS. This difference reflects the discrepancy at $0.8^{-}$ $1 \mathrm{keV}$ between the EPN and the EMOS shown in Fig. 2. This energy band corresponds to an instrumental lowenergy tail of the Fe-L lines and the effect for the EPN is stronger than that of the EMOS. The EPN has an additional uncertainty due to the position dependent energy resolution. Considering that the EMOS spectra of $R>6^{\prime}$ at $0.7-1.1 \mathrm{keV}$ are well fitted by the single temperature MEKAL model fit, the larger amount of $1 \mathrm{keV}$ component observed by EPN may be caused by the uncertainty of the response matrix of the EPN. Thus, there is a systematic uncertainty of $1-2 \%$ in the fraction of the $1 \mathrm{keV}$ component. In summary, within $R<6^{\prime}$, we detected at least two temperature components and outside $R>6^{\prime}$, the upper limit of the second component is less than a few $\%$ even using the EPN.
ASCA spectra of M 87 are also well fitted with a sum of two Raymond-Smith models (Raymond \& Smith 1977). The temperature of the two components are 3.0 and $1.3 \mathrm{keV}$, and the ratio of the emission measure range from 0.2 to 2 within $10^{\prime}$ (Matsumoto et al. 1996), although the results of MEKAL model fit with ASCA spectra are mostly consistent when considering the difference in spatial resolution (Finoguenov \& Jones 2000; Shibata 2000). The contribution of the cooler component plotted in Fig. 5 is much smaller than that derived from R-S fits.

\subsection{Azimuthal temperature structure}

In a second step, in order to study the spatial distribution of the temperature components in more detail, the EMOS spectra are accumulated within sector regions as 


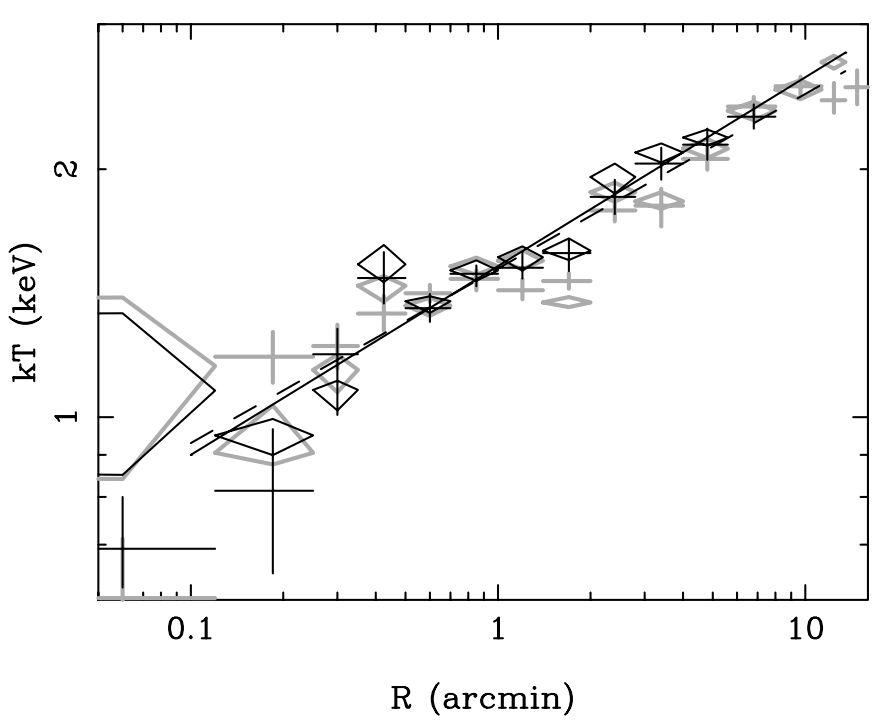

Fig. 3. Deprojected radial profile of the temperature for the EPN (gray crosses) and the EMOS (gray diamonds), obtained using the single temperature MEKAL model. We also plotted the same result but excluding the jet and radio lobe regions (Fig. 1; Sect. 3.3) for the EPN (black crosses) and the EMOS (black diamonds). The solid line and the dashed line corresponds to the best-fit power-law relation (excluding the lobe regions) for the EMOS and EPN, respectively.

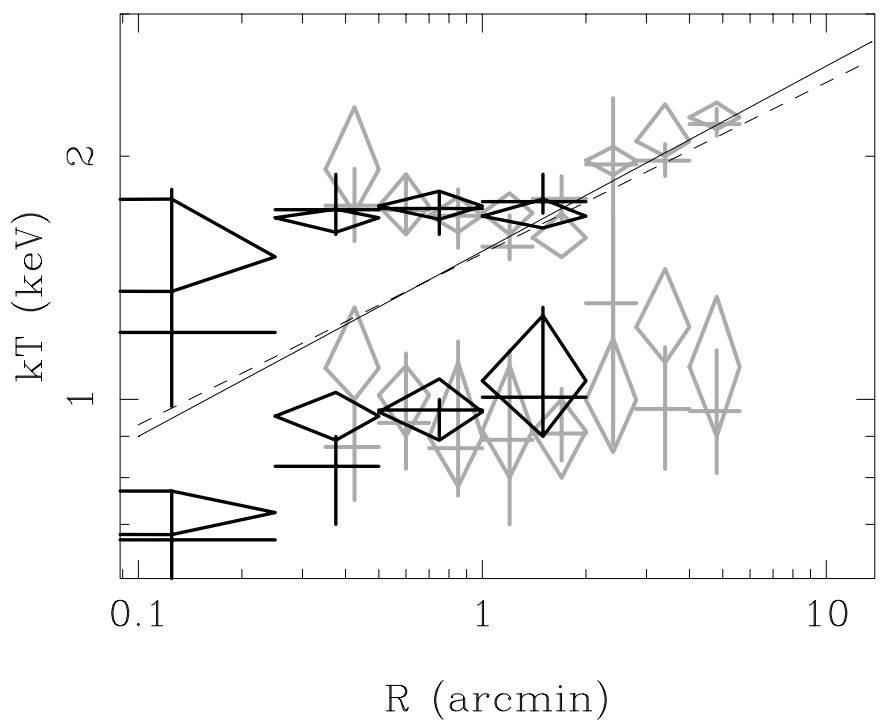

Fig. 4. The radial temperature profile obtained by fitting the spectra with a two component MEKAL model. The meanings of symbols is the same as in Fig. 3. The solid (EMOS) and dashed line (EPN) corresponds to the best-fit power-law ration of the single component MEKAL fit, excluding the radio lobe regions.

shown in Fig. 6. The spectral fits used two temperature MEKAL model. Although the spectra fitted in this section are not the deprojected ones, we can constrain the spatial distribution of the temperature structure. Figure 6 shows EMOS images of the Fe-L $(0.6-1.2 \mathrm{keV})$ and hard (2$10 \mathrm{keV}$ ) energy band with the temperatures obtained for the two components. It also shows hardness ratio maps for the energy band ratios $2-10 \mathrm{keV} / 0.6-1.2 \mathrm{keV}$ and

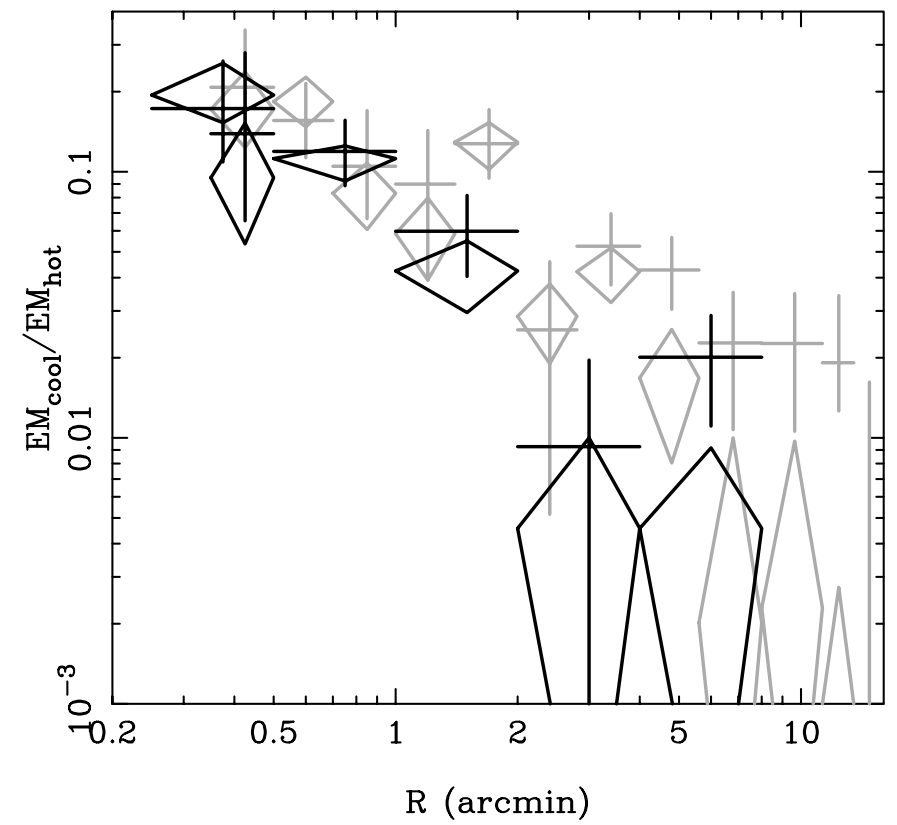

Fig. 5. The ratio of emission measure of the cool and hot temperature component, assuming the temperature of the former to be $1.0 \mathrm{keV}$. The meanings of symbols is the same as in Fig. 3. The discrepancies between the EPN and EMOS may be caused by instrumental problems in the EPN (see text).

1.0-1.2 keV/0.8-1.0 keV. Although the latter is the most sensitive to the $1 \mathrm{keV}$ components (Fig. 7), the statistics is not sufficient in the outer regions. The ratios of the emission measure of the cool to hot components are overlayed on the hardness ratio maps.

The hard band image is nearly spherically symmetric and no enhancements of the X-ray emission associated with the radio structure is seen. The temperature of the hot components is also almost spherically symmetric within $10 \%$. It tends to be slightly lower in the region around the radio lobes.

In contrast, the spatial distribution of the cool component is far from symmetry, although its temperature is determined to have an almost constant value, $\sim 1 \mathrm{keV}$. Belsole et al. (2001) found that the softer regions agree well with the radio structures. Within $R<1^{\prime}$, the $1 \mathrm{keV}$ component is detected from all the spectra, but its contribution ranges from several $\%$ to $30 \%$. In the radial zone $R=1-2^{\prime}$, where 4 sectors are located outside the radio lobes, we find a signature of the $1 \mathrm{keV}$ component in two of these sectors but it is absent in the other two. Outside $2^{\prime}$, we have only detected the $1 \mathrm{keV}$ component from the two sectors containing the radio lobes.

\subsection{Excluding the radio lobe regions}

For the further analysis, we have filtered regions with excess emission related to the jet and radio lobes. We used a spatial filter, excluding those regions where the brightness is larger by $15 \%$ than the azimuthally averaged value (Figs. 1, 6). The softer regions are not fully excluded 

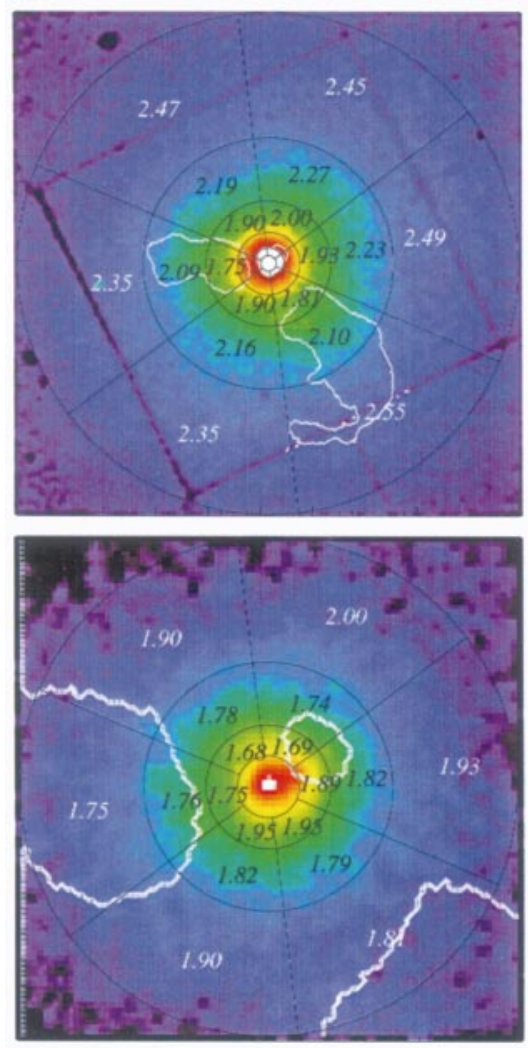
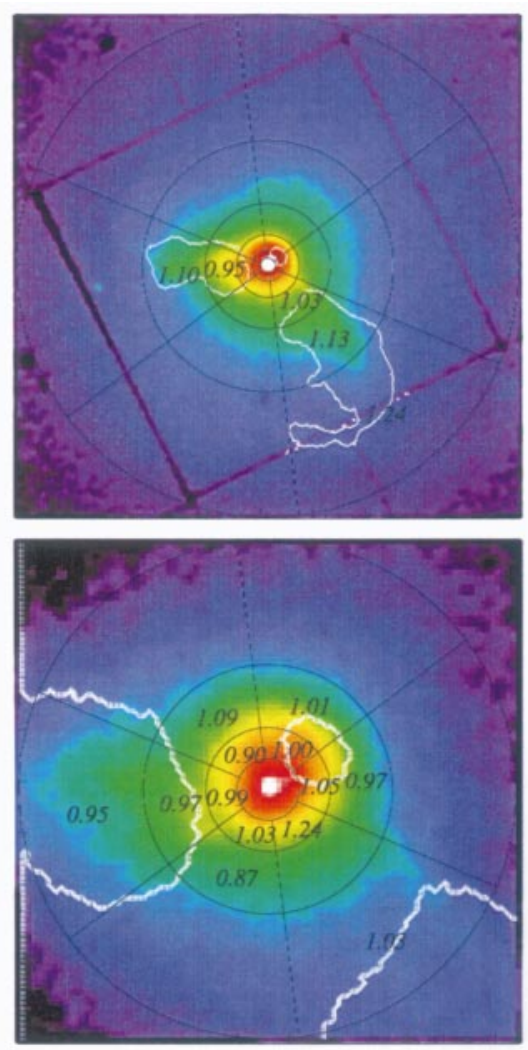
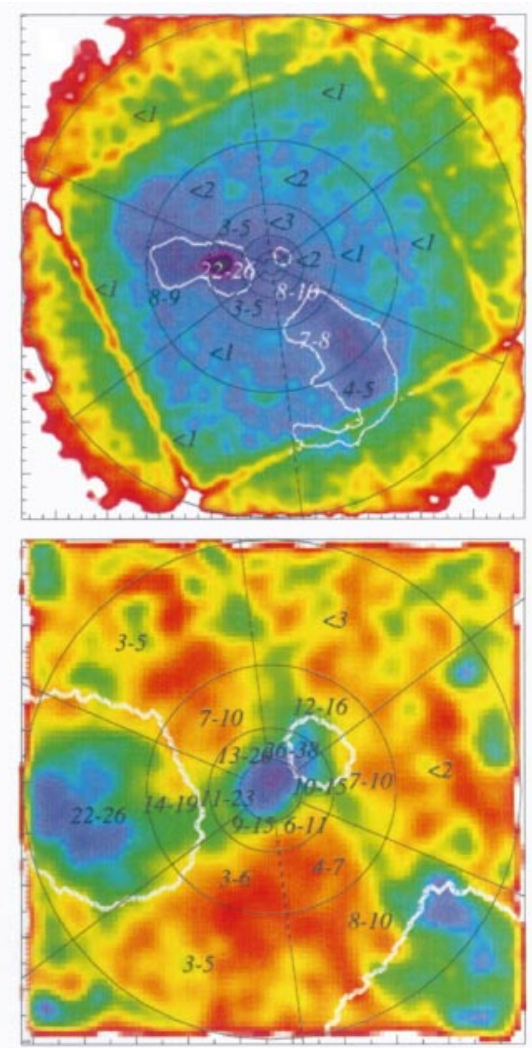

Fig. 6. Hard (2-10 keV; left panels) and Fe-L blend (0.6-1.2 keV; middle panels) EMOS images, overlayed by the hard and soft components temperatures of the two MEKAL component model fit to the projected sector regions, respectively. The $90 \%$ uncertainty of temperatures of the cool and hot components are typically 0.1 and $0.03 \mathrm{keV}$, respectively. The top panels and bottom panels are $16^{\prime} \times 16^{\prime}$ and $4^{\prime} \times 4^{\prime}$ regions, respectively. The right panels show the hardness ratio maps of $2-10 \mathrm{keV} / 0.6-$ $1.2 \mathrm{keV}$ (top) and 1.0-1.2 keV/0.8-1.0 keV (bottom), overlayed by the ratio of the emission measure of the soft to hard components in unit of percent. White contours corresponds to the regions excluded from spectral analysis after Sect. 3.3.

within $2^{\prime}$, since it is difficult to filter whole the $1 \mathrm{keV}$ component within $2^{\prime}$ due to its complicated structure and the limited spatial resolution of the XMM telescope.

Assuming spherical symmetry, deprojected spectra are calculated. We also made deprojected spectra with larger shell width than those in Table 1 . We accumulated spectra within annular regions again and subtracted the contribution from the outer shells with thin shells width obtained before. We then fitted the spectra with the single temperature and two temperature MEKAL model, in the same way as in Sect. 3.1. In the following sections, we will only use the spectra filtered the regions with enhance emissions.

The results are summarized in Table 2. After filtering the regions with excess emissions, the emission measure of the $1 \mathrm{keV}$ component is reduced (Fig. 5), and the single temperature model gives reasonable fits at $R>2^{\prime}$ (Table 2; Fig. 7). The remaining $1 \mathrm{keV}$ components of the EPN beyond $2^{\prime}$ may be due to the uncertainty of the instrumental low-energy tail of the Fe-L lines. The radial temperature profile from the single temperature MEKAL model fit is approximated as $k T=1.52_{-0.02}^{+0.02} R^{0.23_{-0.01}^{+0.01}} \mathrm{keV}$ for the EMOS and $k T=1.51_{-0.03}^{+0.03} R^{0.21_{-0.01}^{+0.02}} \mathrm{keV}$ for the EPN (Fig. 3), where $R$ is in units of arcmin.
Within $R<2^{\prime}$, the two temperature model still gives better fits than the single temperature model, because of the complex structure of the $1 \mathrm{keV}$ within the region. When we adopted the hotter component temperature within $2^{\prime}$, the radial temperature profile is approximated as $k T=1.69_{-0.08}^{+0.09}\left(1+\left(R / 1.6^{\prime}\right)\right)^{0.115_{-0.010}^{+0.011}} \mathrm{keV}$ and $k T=1.68_{-0.05}^{+0.04}\left(1+\left(R / 1.6^{\prime}\right)\right)^{0.100_{-0.016}^{+0.017}} \mathrm{keV}$ for the EMOS and $\mathrm{EPN}$, respectively.

There are some residuals at $\sim 1.2 \mathrm{keV}$, which are common to all the deprojected shells. Any multi-temperature model cannot explain the residual, because the continuum and the Fe-K line are fitted well with a single MEKAL model. When the Ni abundance is assumed to be a factor of 2 larger than Fe in solar units, the residuals become smaller (Finoguenov et al. 2002; Matsushita et al. 2002). This structure may also be due to a problem with the FeL atomic data, since the APEC code (Smith et al. 2001) does not need such a high $\mathrm{Ni}$ abundance.

These results indicate that most of the space is occupied by a component which is nearly spherically symmetrical. Within relatively small regions associated to the radio structures, there is an additional component whose temperature is about $1 \mathrm{keV}$. In the following sections, we 
Table 2. Result of spectrum fitting of the deprojected spectra (regions with the enhanced X-ray emission associated to the radio structures are excluded).

\begin{tabular}{|c|c|c|c|c|c|c|c|c|c|}
\hline \multicolumn{5}{|c|}{ 1T MEKAL } & \multicolumn{5}{|c|}{$2 \mathrm{~T}$ MEKAL } \\
\hline $\begin{array}{r}R \\
(\operatorname{arcmin})\end{array}$ & & $\begin{array}{c}k T \\
(\mathrm{keV})\end{array}$ & $\begin{array}{c}N_{\mathrm{H}} \\
\left(10^{20} \mathrm{~cm}^{-2}\right)\end{array}$ & $\chi^{2} / \mu^{a}$ & $\begin{array}{r}k T 1 \\
(\mathrm{keV})\end{array}$ & $\begin{array}{c}k T 2 \\
(\mathrm{keV})\end{array}$ & $\begin{array}{c}N_{\mathrm{H}} \\
\left(10^{20} \mathrm{~cm}^{-2}\right)\end{array}$ & $\chi^{2} / \mu$ & $\mathrm{EM}_{2} / \mathrm{EM}^{b}{ }^{b}$ \\
\hline $0.00-0.12$ & $\mathrm{EPN}$ & $0.69_{-0.07}^{+0.11}$ & 2.0 (fix) & $49 / 56$ & & & & & \\
\hline $0.12-0.25$ & $\mathrm{EPN}$ & $0.81_{-0.17}^{+0.15}$ & $9.5_{-4.3}^{+7.5}$ & $35 / 54$ & & & & & \\
\hline $0.25-0.35$ & $\mathrm{EPN}$ & $1.19_{-0.19}^{+0.09}$ & $3.0_{-2.7}^{+5.9}$ & $72 / 54$ & & & & & \\
\hline $0.35-0.50$ & $\mathrm{EPN}$ & $1.48_{-0.10}^{+0.11}$ & $4.7_{-2.6}^{+3.0}$ & $65 / 54$ & $1.77_{-0.22}^{+0.26}$ & $0.89_{-0.17}^{+0.22}$ & $2.8_{-2.8}^{+3.4}$ & $55 / 52$ & $0.139_{-0.072}^{+0.142}$ \\
\hline $0.50-0.70$ & $\mathrm{EPN}$ & $1.36_{-0.05}^{+0.05}$ & $5.7_{-1.7}^{+2.7}$ & $67 / 54$ & $1.71_{-0.13}^{+0.17}$ & $0.84_{-0.10}^{+0.17}$ & $2.7_{-2.6}^{+2.6}$ & $46 / 52$ & $0.180_{-0.068}^{+0.116}$ \\
\hline $0.70-1.00$ & $\mathrm{EPN}$ & $1.49_{-0.05}^{+0.03}$ & $4.4_{-1.8}^{+1.7}$ & $43 / 54$ & $1.72_{-0.17}^{+0.20}$ & $1.05_{-0.22}^{+0.26}$ & $2.3_{-2.3}^{+2.3}$ & $35 / 52$ & $0.085_{-0.043}^{+0.055}$ \\
\hline $1.00-1.40$ & $\mathrm{EPN}$ & $1.52_{-0.04}^{+0.07}$ & $4.4_{-1.9}^{+1.6}$ & $35 / 54$ & $1.64_{-0.11}^{+0.15}$ & $0.91_{-0.26}^{+0.33}$ & $3.3_{-2.0}^{+1.8}$ & $30 / 52$ & $0.059_{-0.039}^{+0.043}$ \\
\hline $1.40-2.00$ & $\mathrm{EPN}$ & $1.58_{-0.08}^{+0.06}$ & $3.4_{-1.7}^{+1.7}$ & $56 / 54$ & $1.82_{-0.18}^{+8.72}$ & $1.12_{-0.27}^{+0.42}$ & $1.7_{-1.7}^{+2.1}$ & $46 / 52$ & $0.060_{-0.030}^{+0.040}$ \\
\hline $2.00-2.80$ & $\mathrm{EPN}$ & $1.85_{-0.09}^{+0.09}$ & $2.0_{-1.2}^{+1.2}$ & $35 / 54$ & & & & & $0.008_{-0.008}^{+0.020}$ \\
\hline $2.80-4.00$ & $\mathrm{EPN}$ & $2.03_{-0.09}^{+0.09}$ & $2.2_{-0.9}^{+1.0}$ & $70 / 54$ & & & & & $0.011_{-0.011}^{+0.016}$ \\
\hline $4.00-5.60$ & $\mathrm{EPN}$ & $2.14_{-0.09}^{+0.10}$ & $1.9_{-1.1}^{+0.8}$ & $74 / 54$ & $2.20_{-0.11}^{+0.12}$ & 1.0 (fix) & $1.5_{-1.0}^{+1.0}$ & $65 / 53$ & $0.018_{-0.015}^{+0.015}$ \\
\hline $0.0-0.25$ & $\mathrm{EPN}$ & $0.87_{-0.05}^{+0.07}$ & $5.8_{-2.8}^{+1.2}$ & $53 / 54$ & $1.21_{-0.23}^{+0.61}$ & $0.67_{-0.36}^{+0.10}$ & $2.0_{-2.0}^{+10.2}$ & $31 / 53$ & $3.689_{-3.233}^{+11.461}$ \\
\hline $0.25-0.50$ & $\mathrm{EPN}$ & $1.35_{-0.06}^{+0.06}$ & $5.5_{-1.7}^{+1.9}$ & $76 / 54$ & $1.66_{-0.13}^{+0.15}$ & $0.82_{-0.09}^{+0.10}$ & $3.1_{-2.3}^{+2.0}$ & $47 / 51$ & $0.173_{-0.064}^{+0.088}$ \\
\hline $0.50-1.00$ & $\mathrm{EPN}$ & $1.47_{-0.04}^{+0.02}$ & $4.5_{-1.0}^{+1.6}$ & $84 / 54$ & $1.72_{-0.10}^{+0.11}$ & $0.97_{-0.12}^{+0.13}$ & $2.3_{-1.4}^{+1.4}$ & $49 / 52$ & $0.119_{-0.030}^{+0.040}$ \\
\hline $1.00-2.00$ & $\mathrm{EPN}$ & $1.55_{-0.04}^{+0.05}$ & $4.1_{-1.1}^{+0.9}$ & $71 / 54$ & $1.74_{-0.09}^{+0.16}$ & $1.03_{-0.17}^{+0.26}$ & $2.3_{-1.2}^{+1.2}$ & $51 / 52$ & $0.060_{-0.020}^{+0.022}$ \\
\hline $2.00-4.00$ & $\mathrm{EPN}$ & $1.97_{-0.05}^{+0.06}$ & $2.2_{-0.6}^{+0.6}$ & $91 / 54$ & & & & & $0.009_{-0.009}^{+0.010}$ \\
\hline $0.00-0.12$ & EMOS & $1.08_{-0.23}^{+0.26}$ & $3.4_{-2.4}^{+2.2}$ & $113.6 / 120$ & & & & & \\
\hline $0.12-0.25$ & EMOS & $0.95_{-0.05}^{+0.05}$ & $4.6_{-0.9}^{+1.0}$ & $130.9 / 120$ & & & & & \\
\hline $0.25-0.35$ & EMOS & $1.08_{-0.06}^{+0.03}$ & $4.8_{-2.0}^{+2.1}$ & $107.5 / 120$ & & & & & \\
\hline $0.35-0.50$ & EMOS & $1.53_{-0.07}^{+0.09}$ & $4.0_{-1.6}^{+1.6}$ & $117.6 / 116$ & $1.83_{-0.20}^{+0.33}$ & $1.02_{-0.16}^{+0.28}$ & $0.9_{-0.4}^{+1.6}$ & $100.4 / 114$ & $0.098_{-0.051}^{+0.079}$ \\
\hline $0.50-0.70$ & EMOS & $1.38_{-0.04}^{+0.02}$ & $4.4_{-0.7}^{+1.1}$ & $149.0 / 116$ & $1.83_{-0.13}^{+0.27}$ & $1.08_{-0.08}^{+0.12}$ & $2.1_{-1.3}^{+1.3}$ & $111.1 / 114$ & $0.179_{-0.040}^{+0.050}$ \\
\hline $0.70-1.00$ & EMOS & $1.51_{-0.04}^{+0.04}$ & $3.9_{-1.4}^{+0.7}$ & $118.1 / 116$ & $1.71_{-0.11}^{+0.09}$ & $0.90_{-0.10}^{+0.20}$ & $2.0_{-1.0}^{+1.1}$ & $93.0 / 114$ & $0.088_{-0.024}^{+0.028}$ \\
\hline $1.00-1.40$ & EMOS & $1.56_{-0.06}^{+0.05}$ & $3.4_{-1.2}^{+0.5}$ & $118.0 / 116$ & $1.66_{-0.06}^{+0.14}$ & $0.86_{-0.16}^{+0.34}$ & $2.4_{-1.0}^{+1.0}$ & $110.0 / 114$ & $0.050_{-0.021}^{+0.023}$ \\
\hline $1.40-2.00$ & EMOS & $1.59_{-0.04}^{+0.06}$ & $2.9_{-0.9}^{+0.9}$ & $103.3 / 116$ & $1.68_{-0.06}^{+0.08}$ & 1.0 (fix) & $2.1_{-1.0}^{+1.0}$ & 99.5/114 & $0.035_{-0.020}^{+0.021}$ \\
\hline $2.00-2.80$ & EMOS & $1.96_{-0.09}^{+0.07}$ & $1.9_{-0.8}^{+0.6}$ & $112.4 / 116$ & & & & & $0.019_{-0.010}^{+0.011}$ \\
\hline $2.80-4.00$ & EMOS & $2.10_{-0.06}^{+0.05}$ & $1.2_{-0.4}^{+0.5}$ & $121.3 / 116$ & & & & & $0.000_{-0.000}^{+0.007}$ \\
\hline $4.00-5.60$ & EMOS & $2.19_{-0.05}^{+0.05}$ & $1.1_{-0.4}^{+0.4}$ & $116.4 / 116$ & & & & & $0.008_{-0.008}^{+0.008}$ \\
\hline $0.00-0.25$ & EMOS & $0.95_{-0.09}^{+0.09}$ & $4.0_{-0.7}^{+0.6}$ & $176.9 / 120$ & $1.50_{-0.10}^{+0.30}$ & $0.72_{-0.02}^{+0.08}$ & $2.2_{-1.0}^{+0.7}$ & $104.2 / 114$ & $0.488_{-0.276}^{+2.953}$ \\
\hline $0.25-0.50$ & EMOS & $1.37_{-0.04}^{+0.04}$ & $4.6_{-1.0}^{+1.0}$ & $153.6 / 120$ & $1.68_{-0.08}^{+0.02}$ & $0.95_{-0.05}^{+0.05}$ & $2.7_{-0.6}^{+0.7}$ & $113.7 / 114$ & $0.194_{-0.041}^{+0.062}$ \\
\hline $0.50-1.00$ & EMOS & $1.46_{-0.01}^{+0.01}$ & $4.2_{-0.5}^{+0.4}$ & $196.4 / 116$ & $1.74_{-0.04}^{+0.06}$ & $0.96_{-0.06}^{+0.14}$ & $2.0_{-0.7}^{+0.7}$ & $112.4 / 114$ & $0.112_{-0.020}^{+0.013}$ \\
\hline $1.00-2.00$ & EMOS & $1.58_{-0.02}^{+0.01}$ & $3.1_{-0.4}^{+0.5}$ & $150.2 / 116$ & $1.69_{-0.09}^{+0.11}$ & $1.05_{-0.15}^{+0.25}$ & $2.2_{-0.6}^{+0.5}$ & $135.4 / 114$ & $0.042_{-0.013}^{+0.013}$ \\
\hline $2.00-4.00$ & EMOS & $2.05_{-0.04}^{+0.03}$ & $1.5_{-0.4}^{+0.3}$ & $171.8 / 116$ & & & & & $0.005_{-0.005}^{+0.005}$ \\
\hline $4.00-8.00$ & EMOS & $2.30_{-0.04}^{+0.04}$ & $1.6_{-0.2}^{+0.2}$ & $168.2 / 116$ & & & & & $0.005_{-0.005}^{+0.005}$ \\
\hline
\end{tabular}

${ }^{a}$ Degrees of freedom.

${ }^{b}$ Ratio of emission measure of the cooler and hotter component, when the temperature of the former is fixed to $1 \mathrm{keV}$.

will study the temperature structure in detail using the filtered spectra.

\section{The abundance profile of Fe}

In spectral fitting the derived temperature and element abundances are partly dependent on each other. This is particularly important for the Fe abundance, where the spectral features of $\mathrm{K}$ - and L-shell lines with their temperature dependent relative importance are fitted simultaneously. A more detailed report on the abundances is given in Paper II (Matsushita et al. 2002) and Finoguenov et al. (2002), however we briefly discuss here the results on the $\mathrm{Fe}$ abundances in relation to the derived temperature structure. As derived from the projected spectra (Böhringer et al. 2001; Finoguenov et al. 2002), the Fe abundance shows a strong negative gradient (Fig. 8). The two temperature MEKAL fit gives significantly larger abundances than the single temperature MEKAL fit, although the contributions of the cooler component are small. For example, at $R=0.5-1^{\prime}$, including of $10 \%$ of the cooler component in units of emission measure changes $\mathrm{Fe}$ 


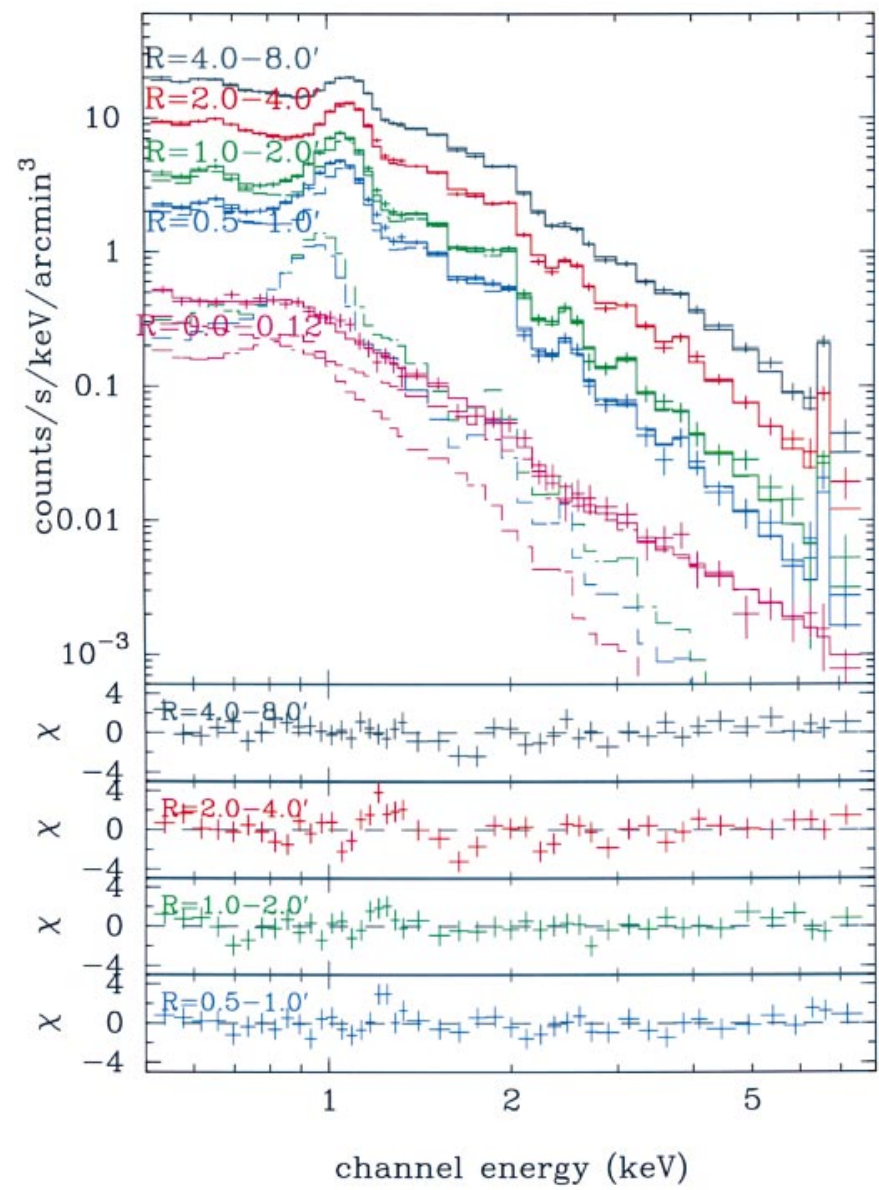

Fig. 7. Deprojected spectra of the EPN (lobes excluded). The spectrum at $R<0.12^{\prime}$ is fitted with the double component model, consisting a MEKAL model and a power-law component and those of $R=0.5-1.0^{\prime}$ and $1.0-2.0^{\prime}$ are fitted with a two component MEKAL model. Each component is plotted in dashed lines. The other spectra are fitted with a MEKAL model. The bottom panels show residuals of the fit.

abundances by a factor of 2 . The reason for this is the change of the temperature of the hot component, when the cold component is added, e.g. in the ring taken as an example, the temperature of the main component increases from 1.47 to $1.72 \mathrm{keV}$. For a fixed Fe line feature this leads to a higher abundance required in the fit. As a result, the central abundance drop that is seen in the single MEKAL fit is not seen in the result of the two MEKAL fit. This will be discussed further in Paper II (Matsushita et al. 2002).

\section{Detailed temperature structure}

\subsection{Temperatures obtained from the Fe-L and the hard energy band}

There are some problems in the determination of the temperature through X-ray spectral fitting. The first problem is resonant line scattering, which should be important in the core of M 87 (Shigeyama 1998; Böhringer et al. 2001). The second problem is the uncertainties in the Fe-L atomic

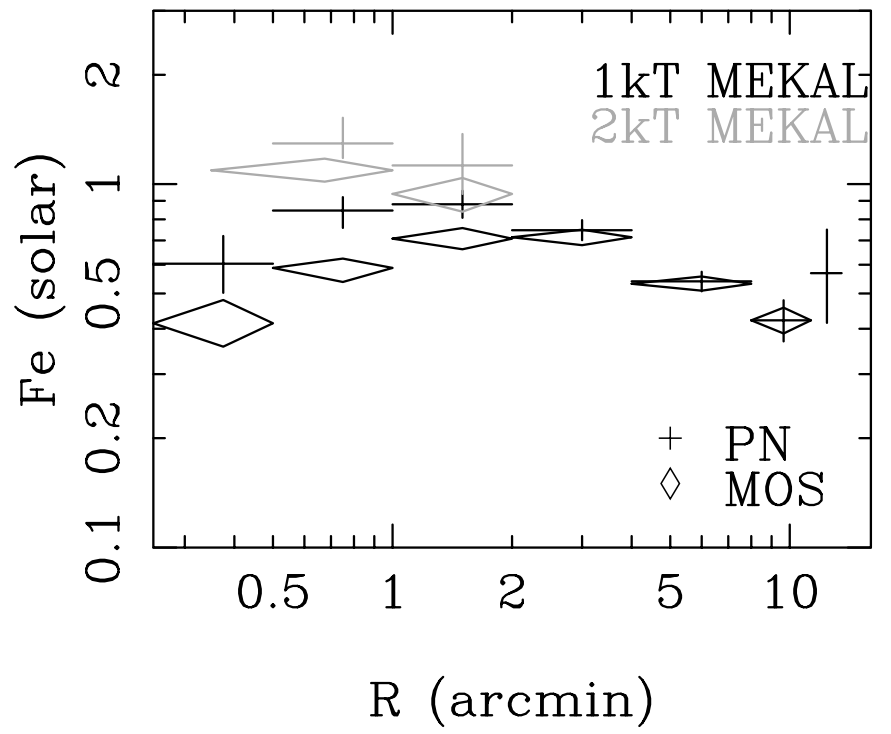

Fig. 8. Deprojected Fe profile of the EPN (crosses) and EMOS (diamonds) fitted with a single temperature MEKAL model (black) and the two temperature MEKAL model (gray).

data (e.g. Masai 1977; Matsushita et al. 2000). The major uncertainty is the ionization and recombination rates of $\mathrm{Fe}$, which may change resulting temperature by $20-30 \%$. (Masai 1997; Matsushita et al. 2000; Arnaud \& Rothenflug 1985; Arnaud \& Raymond 1992). In order to avoid these problems, we exclude the Fe-L region below $1.6 \mathrm{keV}$ and fit the spectra again. In addition, to compare the temperature obtained to those from the Fe-L lines, we also fitted the data between 0.7 to $1.3 \mathrm{keV}$ with a single temperature MEKAL or Raymond-Smith model (hereafter R-S model). The R-S model is used for the comparison with the ASCA results.

The result is summarized in Table 3 and Fig. 9. The derived temperatures using the hard energy band, $k T_{\text {hard }}$, of the EPN and EMOS are consistent with each other (Table 3 ). At $R>2^{\prime}$, they are consistent to those obtained using the whole energy band and within $R=0.35-2^{\prime}$ they are consistent to the temperatures of the hotter component obtained in Sects. 3.1 and 3.3. This indicates that within $R=0.35-2^{\prime}$, the $1.7 \mathrm{keV}$ component really exists and is the dominant phase in the ICM.

The results for the Fe-L region using the MEKAL model, $k T_{\text {MEKAL }}$, are consistent or slightly higher than $k T_{\text {hard }}$ at $R>1^{\prime}$ because of the residual structure around $\sim 1.2 \mathrm{keV}$. Within $R<1^{\prime}, k T_{\text {MEKAL }}$ is lower than $k T_{\text {hard }}$ due to the contributions from the $1 \mathrm{keV}$ components.

The R-S model gives significantly lower temperatures than $k T_{\text {hard }}$ by $\sim 20 \%$. As a result, when fitted with a R-S model, there remain residuals in the hard energy band. To explain the residuals, we need an additional R-S component with a high temperature, $\sim 3 \mathrm{keV}$. This is the reason of the comparable emission measure of the two temperature R-S components obtained in the ASCA results (Matsumoto et al. 1996) 
Table 3. Result of spectrum fitting of the deprojected spectra (radio lobes excluded).

\begin{tabular}{|c|c|c|c|c|}
\hline $\begin{array}{c}R \\
(\operatorname{arcmin})\end{array}$ & $\begin{array}{c}\mathrm{EPN} \\
k T_{\text {hard }}{ }^{a} \\
(\mathrm{keV})\end{array}$ & $\begin{array}{c}\text { EMOS } \\
k T_{\text {hard }}{ }^{a} \\
(\mathrm{keV})\end{array}$ & $\begin{array}{c}k T_{\text {MEKAL }}{ }^{b} \\
(\mathrm{keV})\end{array}$ & $\begin{array}{c}k T_{\mathrm{R}-\mathrm{S}}{ }^{b} \\
(\mathrm{keV})\end{array}$ \\
\hline $0.00-0.12$ & & & $1.08_{-0.27}^{+0.37}$ & $1.01_{-0.02}^{+0.04}$ \\
\hline $0.12-0.25$ & & & $1.03_{-0.05}^{+0.05}$ & $1.01_{-0.03}^{+0.03}$ \\
\hline $0.25-0.35$ & $1.06_{-0.38}^{+0.34}$ & $1.60_{-0.36}^{+0.39}$ & $1.11_{-0.06}^{+0.06}$ & $1.06_{-0.04}^{+0.03}$ \\
\hline $0.35-0.50$ & $1.78_{-0.38}^{+0.52}$ & $1.64_{-0.17}^{+0.18}$ & $1.59_{-0.09}^{+0.11}$ & $1.38_{-0.05}^{+0.04}$ \\
\hline $0.50-0.70$ & $1.61_{-0.15}^{+0.16}$ & $1.68_{-0.11}^{+0.11}$ & $1.41_{-0.04}^{+0.03}$ & $1.24_{-0.07}^{+0.03}$ \\
\hline $0.70-1.00$ & $1.59_{-0.16}^{+0.18}$ & $1.60_{-0.10}^{+0.10}$ & $1.55_{-0.05}^{+0.07}$ & $1.33_{-0.03}^{+0.04}$ \\
\hline $1.00-1.40$ & $1.68_{-0.17}^{+0.18}$ & $1.55_{-0.10}^{+0.10}$ & $1.68_{-0.09}^{+0.08}$ & $1.39_{-0.03}^{+0.02}$ \\
\hline $1.40-2.00$ & $1.74_{-0.15}^{+0.21}$ & $1.58_{-0.10}^{+0.10}$ & $1.73_{-0.10}^{+0.09}$ & $1.39_{-0.03}^{+0.03}$ \\
\hline $2.00-2.80$ & $1.89_{-0.14}^{+0.15}$ & $1.98_{-0.09}^{+0.09}$ & $2.09_{-0.15}^{+0.16}$ & $1.45_{-0.02}^{+0.12}$ \\
\hline $2.80-4.00$ & $2.12_{-0.13}^{+0.14}$ & $2.05_{-0.07}^{+0.07}$ & $2.10_{-0.12}^{+0.15}$ & $1.53_{-0.09}^{+0.11}$ \\
\hline $4.00-5.60$ & $2.22_{-0.14}^{+0.21}$ & $2.15_{-0.08}^{+0.08}$ & $2.58_{-0.25}^{+0.32}$ & $1.65_{-0.13}^{+0.11}$ \\
\hline $5.60-8.00$ & $2.41_{-0.15}^{+0.16}$ & $2.29_{-0.07}^{+0.12}$ & $2.46_{-0.19}^{+0.23}$ & $1.71_{-0.11}^{+0.09}$ \\
\hline $8.00-11.30$ & $2.74_{-0.19}^{+0.21}$ & $2.47_{-0.09}^{+0.09}$ & $2.80_{-0.28}^{+0.34}$ & $1.81_{-0.11}^{+0.29}$ \\
\hline $11.3-13.50$ & $2.58_{-0.30}^{+0.36}$ & $2.61_{-0.05}^{+0.06}$ & $2.65_{-0.19}^{+0.23}$ & $2.04_{-0.14}^{+0.13}$ \\
\hline $13.5-16.00$ & $2.55_{-0.18}^{+0.20}$ & & & \\
\hline
\end{tabular}

${ }^{a} k T$ obtained by fitting the spectra above $1.6 \mathrm{keV}$.

${ }^{b} k T$ obtained by fitting the spectra between 0.7 to $1.3 \mathrm{keV}$.

\subsection{Temperature obtained from line ratios}

The ratio of He-like $\mathrm{K}_{\alpha}$ to $\mathrm{H}$-like $\mathrm{K}_{\alpha}$ line strengths is sensitive to the gas temperature. Since the energy resolution of the EMOS is better than that of the EPN, we used only EMOS data here. We fitted the spectra within the energy range of $1.8-2.1 \mathrm{keV}$, and 2.3-2.7 keV with a MEKAL model. We denote the temperature obtained from the former energy range as $k T_{\mathrm{Si}}$ and from the latter as $k T_{\mathrm{S}}$. The contribution of Fe-L lines at the energy of the He-like $K_{\alpha}$ line of $\mathrm{Si}$ is considerable, and the He-like line contains several strong components. The result is summarized in Table 4.

Figure 9 also compares the temperatures, $k T_{\mathrm{Si}}$ and $k T_{\mathrm{S}}$. These temperatures agree well within $10 \%$ with the deprojected temperature of the corresponding shell, although $k T_{\mathrm{Si}}$ may have some uncertainty, since around the Si He-K lines, Fe-L and Ni-L lines are also important. Furthermore, there is an instrumental Si edge in the response matrix and a strong instrumental Si line, which may cause the discrepancy between the EPN and EMOS at $2 \mathrm{keV}$ shown in Fig. 2.

We also compare the model and data spectra of the $\mathrm{Si}$ and $\mathrm{S}$ region in Fig. 10. The two temperature R-S model with the same abundance ratio, cannot fit the Si and S line ratios, while a single temperature MEKAL model fits the lines well. In order to explain the observed line ratios with the two component R-S model, the harder spectral component should have an abundance of $\mathrm{Si}$ and $\mathrm{S}$ a factor of $3 \sim 5$ larger than the softer component.

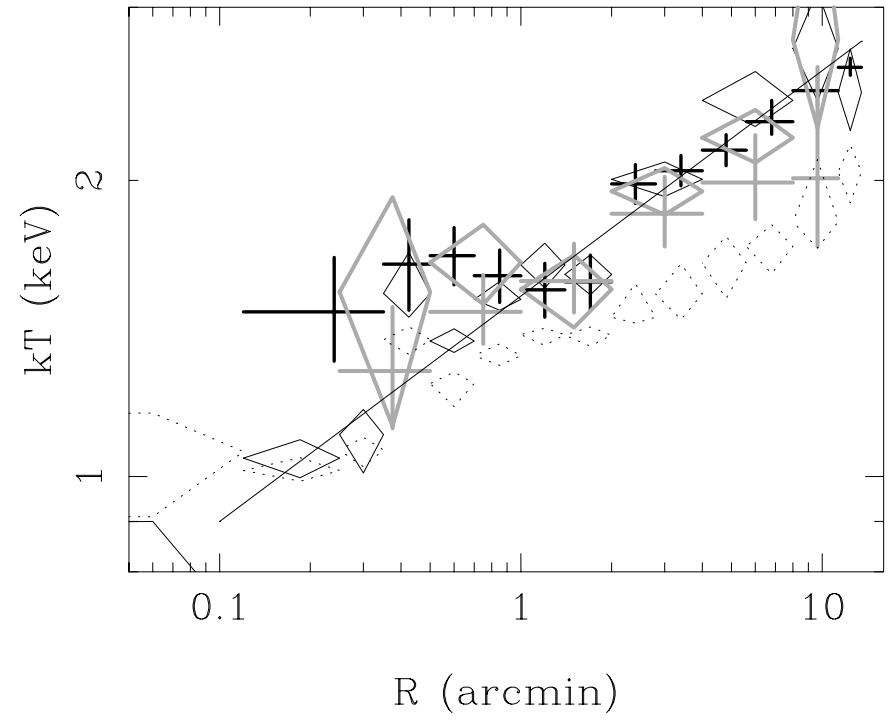

Fig. 9. Deprojected radial profile of the temperature obtained with EMOS, by fitting the spectra of $0.7-1.3 \mathrm{keV}$ (black diamonds), above $1.6 \mathrm{keV}$ (black crosses), 1.8-2.1 keV (gray crosses) and 2.3-2.7 keV (gray diamonds) with a MEKAL model. The results obtained by R-S model using $0.7-1.3 \mathrm{keV}$ are also shown (dotted diamonds). The solid line corresponds to the best fit regression line for the MEKAL model using the whole energy band of the EMOS.

Table 4. Result of spectral fit of EMOS data, using the energy band around the Si and S lines with a MEKAL model.

\begin{tabular}{ccccc}
\hline & $1.8-2.1 \mathrm{keV}$ & $2.3-2.8 \mathrm{keV}$ \\
$R$ & $k T_{\mathrm{Si}}$ & $\chi^{2} / \mu^{a}$ & $k T_{\mathrm{S}}$ & $\chi^{2} / \mu^{a}$ \\
$(\operatorname{arcmin})$ & $(\mathrm{keV})$ & & $(\mathrm{keV})$ & \\
\hline $0.25-0.50$ & $1.28_{-0.16}^{+0.21}$ & $19.2 / 19$ & $1.54_{-0.42}^{+0.38}$ & $18.4 / 31$ \\
$0.50-1.00$ & $1.47_{-0.11}^{+0.13}$ & $9.3 / 19$ & $1.65_{-0.15}^{+0.15}$ & $14.6 / 31$ \\
$1.00-2.00$ & $1.58_{-0.11}^{+0.15}$ & $17.5 / 19$ & $1.55_{-0.13}^{+0.13}$ & $31.2 / 31$ \\
$2.00-4.00$ & $1.85_{-0.14}^{+0.17}$ & $12.8 / 19$ & $1.95_{-0.10}^{+0.11}$ & $20.4 / 31$ \\
$4.00-8.00$ & $1.99_{-0.16}^{+0.23}$ & $9.0 / 19$ & $2.21_{-0.12}^{+0.15}$ & $23.0 / 31$ \\
$8.00-11.30$ & $2.01_{-0.30}^{+0.60}$ & $12.7 / 19$ & $2.78_{-0.52}^{+1.26}$ & $19.9 / 31$ \\
\hline
\end{tabular}

${ }^{a}$ Degrees of freedom.

Since the deprojected data have larger uncertainties, we have also used the $\mathrm{S}$ line ratios of the annular spectra. The strength of the $\mathrm{K} \alpha$ lines are obtained by fitting the annular spectra within $2.2-2.8 \mathrm{keV}$ with a bremsstrahlung and two Gaussians. We calculated the projected S line ratio using the deprojected temperature and density (Sect. 7) profiles and compared with the observed ones. We used two temperature relations derived in Sect. 3.3. One is the best fit relation of the single MEKAL model fit and the other relation is derived when we adopted the hotter component temperature of the two temperature MEKAL fit within $R<2^{\prime}$. Outside $1^{\prime}$, the observed ratios agree well with the calculated profiles from single temperature model fit (Fig. 11). Comparing these results with a profile calculated using a temperature profile shifted by $10 \%$ to the data, we can conclude that the temperature profile inferred from the observed $\mathrm{S}$ line ratios 


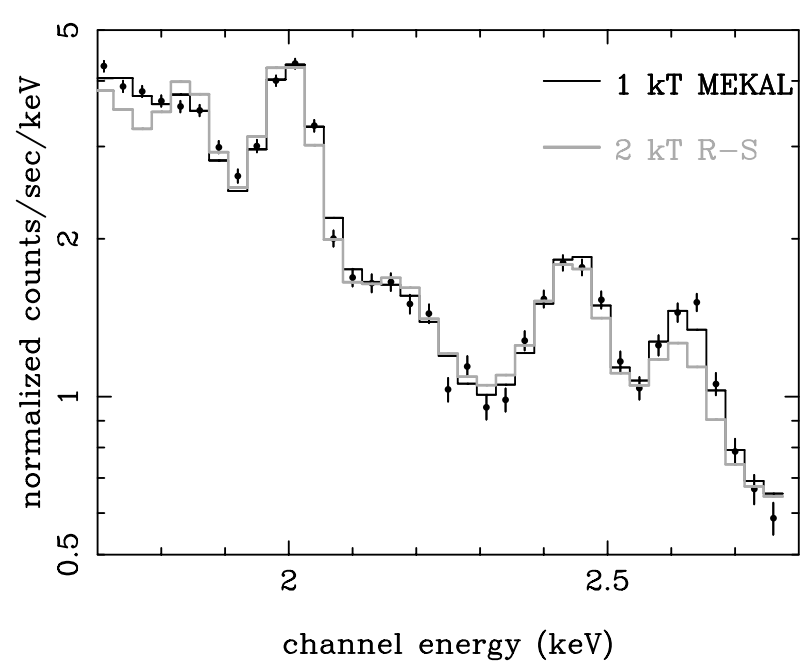

Fig. 10. Deprojected EMOS spectrum within $R=1-2.8^{\prime}$ in the energy range of $\mathrm{Si}$ and $\mathrm{S}$ lines, fitted with a MEKAL model (black line) or $1.4 \mathrm{keV}+3.3 \mathrm{keV}$ R-S model (gray line).

is consistent within several \% with the deprojected temperature profile. Within $1^{\prime}$, the observed ratios are close to with those calculated using the hotter component temperature of the two temperature MEKAL fit, but smaller than those from the single temperature fit. This result indicates that $\mathrm{S}$ lines are dominated by those of the hotter component within $2^{\prime}$.

We then compared the strength of the Fe-L and Fe-K lines as follows. As for the $\mathrm{S}$ line ratios, the line brightness profile of Fe-K is calculated using the deprojected results. Within $2^{\prime}$, we adopted the Fe abundance obtained from the two temperature model fit. The Fe abundance from the deprojected data is mainly determined from the Fe-L lines. For a given temperature, this analysis gives a comparison between the Fe-L and Fe-K spectral signatures. Outside $0.5^{\prime}$, the observed profile of Fe-K is only $20 \%$ larger than the calculated profile (Fig. 12). Since the Fe-L atomic data may have uncertainties of 20-30\% (e.g. Masai et al. 1997; Matsushita et al. 2000), the difference may be due to the uncertainties in the Fe-L atomic data. If the difference is caused by the temperature structure, it is explained by only $10 \%$ larger temperatures.

In summary, excluding the regions associated to the radio structures, at $R>1^{\prime}, k T_{\text {hard }}, k T_{\mathrm{Si}}$ and $k T_{\mathrm{S}}$ of a given deprojected spectrum agree within $10 \%$. $k T_{\text {MEKAL }}$ also agrees well with $k T_{\text {hard }}$, although $k T_{\mathrm{Fe}-\mathrm{L}}$ depends on the plasma emission code (e.g. Masai 1997). These results indicate that at least at $R>1^{\prime}$, the ICM at a given radius is dominated by a single temperature component whose temperature is $1.7 \mathrm{keV}$ at $R=1^{\prime}$ and $2.5 \mathrm{keV}$ at $R=15^{\prime}$. The consistency between Fe-L and Fe-K also supports the single phase temperature structure. The difference of these temperatures within $1^{\prime}$ reflects that the contribution of the $1 \mathrm{keV}$ component become significant.

Moreover, the temperature obtained from the MEKAL model seems to be reasonable at least in the obtained temperature range, when ignoring the residual structure at $1.2 \mathrm{keV}$

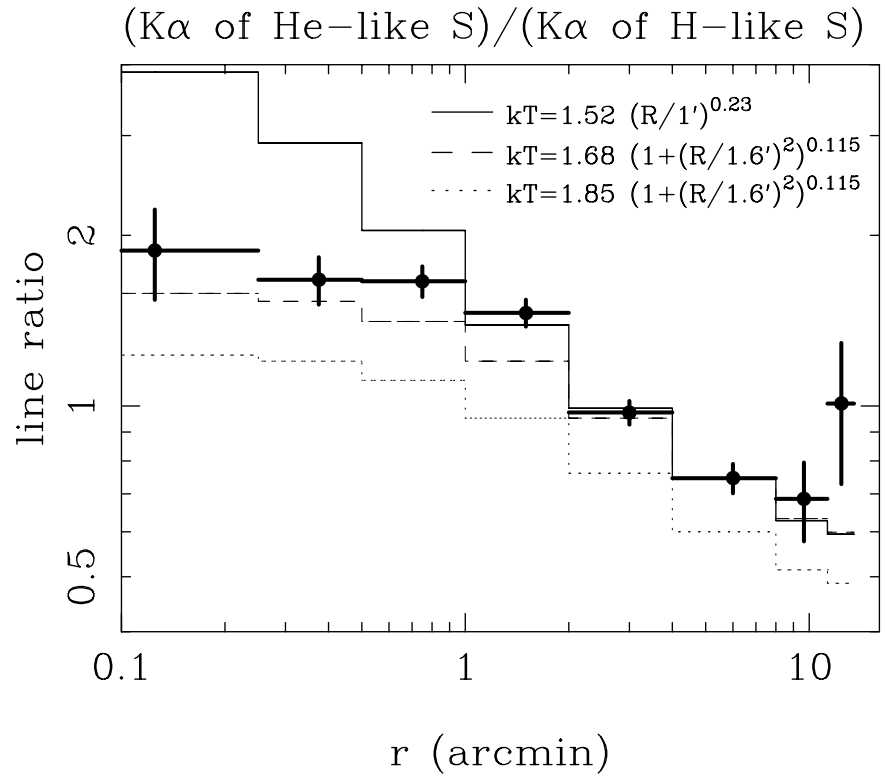

Fig. 11. Projected line ratios (EMOS) between $\mathrm{K} \alpha$ of hydrogen and helium like S (closed circles). Errors correspond to $68 \%$ confidence level. The solid and dashed lines represent the results using the observed temperature profile from the single temperature MEKAL model and that of the hotter component from the two temperature MEKAL model, respectively. The assumed radial temperature profiles are written in the figure. We also plotted the profile for temperatures shifted by $10 \%$ (dotted line).

\subsection{Spectral fits with the APEC plasma code}

A new plasma code, APEC (Smith et al. 2001), is now available in XSPEC. The residual structures around the Fe-L emission that occurred in the fits with the MEKAL code disappeared with the use of the APEC code for Centaurus clusters (Sanders \& Fabian 2001).

In this paper, we used the version 1.0 of the APEC in the version 11.0.1 of the XSPEC. In Fig. 13, we show ratios of a single temperature plasma with solar abundance calculated with the APEC and MEKAL models, when observed with the EMOS. Within the energy resolution of the CCD detectors, the dominant change is not in the Fe-L lines, but in the $\mathrm{K} \alpha$ lines. Using the APEC code, the strength of the $\mathrm{K} \alpha$ lines of hydrogen like ions, which are the most fundamental lines, decrease by several tens of $\%$, and the temperature dependence also changes. The $6.7 \mathrm{keV}$ Fe-K lines decrease, by a factor of 2 at $1 \mathrm{keV}$ and $30 \%$ at $4 \mathrm{keV}$. The changes of the $\mathrm{K} \alpha$ lines of helium like ions of Si, S, Ar and Ca are smaller.

Figure 14 shows a representative EMOS spectrum of M 87 fitted with the MEKAL and APEC model. As already shown, the single temperature MEKAL model can well fit the spectra including the strength of Fe-L, Fe-K and $\mathrm{S}$ lines. The APEC model gives a better fit for the Fe$\mathrm{L} / \mathrm{Mg}-\mathrm{K}$ structure around $1.2-1.5 \mathrm{keV}$ and the Fe-L/Si-K structure around $1.8 \mathrm{keV}$. However, the single temperature APEC model cannot fit the Fe-L and Fe-K, as well 


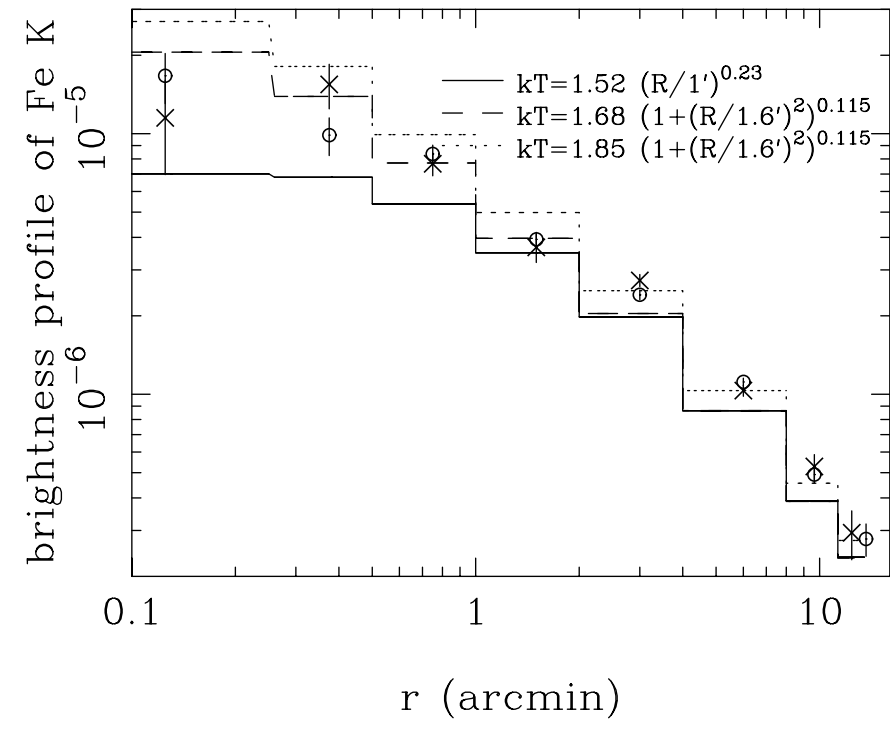

Fig. 12. The projected radial profile of the line brightness of Fe-K $\alpha$ of the EMOS (open circles) and the EPN (crosses). The solid and dashed lines represent the results using the temperature profile from the single temperature MEKAL model and that of the hotter component from the two temperature MEKAL model, respectively. The dotted lines corresponds to $10 \%$ higher temperatures. Errors represent the $68 \%$ confidence level.

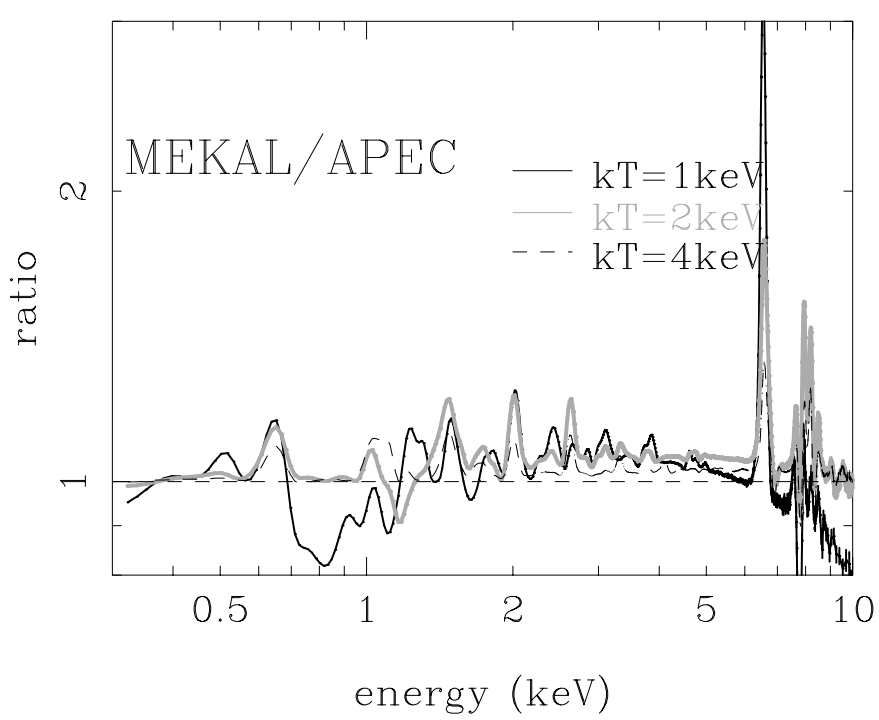

Fig. 13. Ratio of theoretical spectra of MEKAL to APEC code for a plasma temperature of $1 \mathrm{keV}$ (black solid line), $2 \mathrm{keV}$ (gray solid line) and $4 \mathrm{keV}$ (black dashed line) when observed with the EMOS. Abundances are assumed to have solar values.

as the ratio of $\mathrm{K} \alpha$ lines of $\mathrm{S}$ simultaneously, although the fit to the Fe-L complex and the continuum is good.

Using the APEC code, we fitted the deprojected spectra of the EMOS in the same way as described in Sects. 3.3, 5.1 and 5.2. Figure 15 and Tables 5 summarize the temperatures derived with the APEC code. Due to the inconsistency of the $\mathrm{S}$ line ratios and the strength of the Fe-K line, the reduced $\chi^{2}$ is systematically worse at $R>2^{\prime}$ compared to those obtained from the MEKAL model fit.

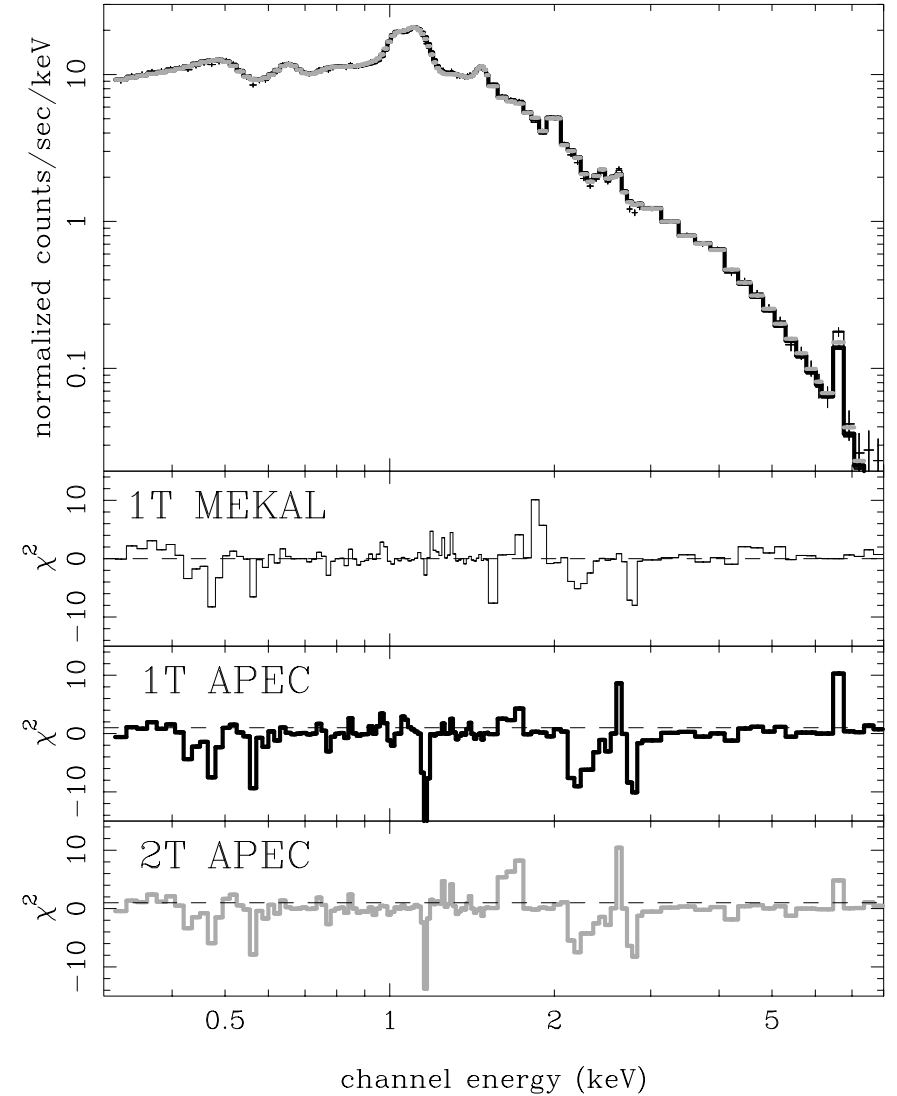

Fig. 14. The deprojected spectrum of EMOS within $R=4-8^{\prime}$ (crosses) fitted with a MEKAL model (thin black lines), an APEC model (bold black lines) and a two temperature APEC model (bold gray lines). The bottom panels shows the contributions to $\chi^{2}$.

The temperatures obtained from the whole energy band, the Fe-L complex and the hard energy band using the APEC code are consistent with those obtained using the MEKAL model. The derived Fe abundances, which are mainly determined by Fe-L lines, are also consistent with those from MEKAL model (Fig. 17). In contrast, the temperatures from Si and S energy band are 10\% larger, due to the changes of the strength of the K $\alpha$ lines of hydrogen like ions.

The projected radial profile of the $\mathrm{S}$ line ratios are also compared with the calculated profile from the deprojected data (Fig. 16). Although the deprojected temperatures cannot reproduce the observed line ratios, the difference is only $10 \%$ in temperature. Therefore, as in the case with MEKAL model, temperatures derived from whole energy band, the Fe-L, the hard band $\mathrm{Si}$, and $\mathrm{S}$ energy band agree within $10 \%$.

However, there is a discrepancy between the Fe-L and Fe-K strength. Figure 17 also shows the Fe abundances obtained from the deprojected spectra above $2 \mathrm{keV}$, fitted with a single temperature MEKAL and APEC model. We denote the derived Fe abundances from $>2 \mathrm{keV}$, as Fe-K $\mathrm{K}_{\mathrm{MEKAL}}$ and Fe- $\mathrm{K}_{\mathrm{APEC}}$, and those from the whole energy band as Fe- $\mathrm{L}_{\text {MEKAL }}$ and Fe- $\mathrm{L}_{\mathrm{APEC}}$ for the MEKAL and APEC codes, respectively. Outside $R>1^{\prime}$, 
Table 5. Result of EMOS spectrum fitting of the deprojected spectra using a APEC model (lobe excluded).

\begin{tabular}{|c|c|c|c|c|c|c|c|}
\hline $\begin{array}{c}R \\
(\operatorname{arcmin})\end{array}$ & $\begin{array}{c}k T \\
(\mathrm{keV})\end{array}$ & $\begin{array}{c}0.3-10 \mathrm{keV} \\
N_{\mathrm{H}} \\
10^{20} \mathrm{~cm}^{-2}\end{array}$ & $\chi^{2} / \mu^{a}$ & $\begin{array}{c}0.7-1.3 \mathrm{keV} \\
k T \\
(\mathrm{keV})\end{array}$ & $\begin{array}{c}1.6-10 \mathrm{keV} \\
k T \\
(\mathrm{keV})\end{array}$ & $\begin{array}{c}1.8-2.1 \mathrm{keV} \\
k T_{\mathrm{Si}} \\
(\mathrm{keV})\end{array}$ & $\begin{array}{c}2.3-2.8 \mathrm{keV} \\
k T_{\mathrm{S}} \\
(\mathrm{keV})\end{array}$ \\
\hline $0.00-0.12$ & $1.04_{-0.15}^{+0.20}$ & $1.6_{-1.0}^{+1.1}$ & $111.9 / 116$ & $1.05_{-0.35}^{+0.25}$ & $1.36_{-0.30}^{+0.98}$ & & \\
\hline $0.12-0.25$ & $1.02_{-0.08}^{+0.01}$ & $4.0_{-0.7}^{+0.6}$ & $140.6 / 116$ & $1.05_{-0.06}^{+0.02}$ & $1.29_{-0.31}^{+0.29}$ & & \\
\hline $0.25-0.35$ & $1.02_{-0.05}^{+0.08}$ & $6.6_{-2.2}^{+1.9}$ & $106.1 / 116$ & $1.06_{-0.08}^{+0.11}$ & $1.53_{-0.50}^{+0.42}$ & & \\
\hline $0.35-0.50$ & $1.61_{-0.10}^{+0.03}$ & $3.6_{-1.9}^{+1.9}$ & $109.0 / 116$ & $1.59_{-0.09}^{+0.09}$ & $1.71_{-0.18}^{+0.24}$ & & \\
\hline $0.50-0.70$ & $1.34_{-0.02}^{+0.01}$ & $5.0_{-0.8}^{+1.8}$ & $148.3 / 116$ & $1.35_{-0.02}^{+0.01}$ & $1.80_{-0.11}^{+0.13}$ & & \\
\hline $0.70-1.00$ & $1.54_{-0.02}^{+0.03}$ & $3.4_{-0.6}^{+1.3}$ & $120.3 / 116$ & $1.58_{-0.04}^{+0.04}$ & $1.73_{-0.09}^{+0.13}$ & & \\
\hline $1.00-1.40$ & $1.62_{-0.05}^{+0.02}$ & $3.3_{-0.8}^{+1.0}$ & $111.0 / 116$ & $1.63_{-0.04}^{+0.04}$ & $1.71_{-0.09}^{+0.10}$ & & \\
\hline $1.40-2.00$ & $1.63_{-0.03}^{+0.01}$ & $1.9_{-0.8}^{+1.0}$ & $92.8 / 116$ & $1.64_{-0.04}^{+0.02}$ & $1.72_{-0.09}^{+0.07}$ & & \\
\hline $2.00-2.80$ & $1.92_{-0.02}^{+0.03}$ & $1.8_{-0.4}^{+0.4}$ & $124.1 / 116$ & $1.88_{-0.08}^{+0.10}$ & $2.06_{-0.07}^{+0.08}$ & & \\
\hline $2.80-4.00$ & $2.12_{-0.03}^{+0.01}$ & $1.1_{-0.3}^{+0.3}$ & $157.1 / 116$ & $1.92_{-0.09}^{+0.07}$ & $2.13_{-0.06}^{+0.06}$ & & \\
\hline $4.00-5.60$ & $2.13_{-0.03}^{+0.04}$ & $1.0_{-0.3}^{+0.3}$ & $123.3 / 116$ & $2.09_{-0.07}^{+0.05}$ & $2.20_{-0.06}^{+0.07}$ & & \\
\hline $5.60-8.00$ & $2.32_{-0.05}^{+0.02}$ & $1.8_{-0.4}^{+0.2}$ & $162.5 / 116$ & $2.10_{-0.08}^{+0.05}$ & $2.35_{-0.07}^{+0.09}$ & & \\
\hline $8.00-11.3$ & $2.54_{-0.06}^{+0.07}$ & $1.7_{-0.3}^{+0.4}$ & $169.4 / 116$ & $2.44_{-0.26}^{+0.17}$ & $2.48_{-0.08}^{+0.09}$ & & \\
\hline $11.3-13.5$ & $2.67_{-0.03}^{+0.04}$ & $1.3_{-0.3}^{+0.1}$ & $308.6 / 116$ & $2.60_{-0.17}^{+0.10}$ & $2.59_{-0.05}^{+0.03}$ & & \\
\hline $0.00-0.25$ & $1.04_{-0.06}^{+0.01}$ & $3.2_{-0.4}^{+0.6}$ & $180.9 / 116$ & $1.02_{-0.04}^{+0.04}$ & $1.52_{-0.27}^{+0.21}$ & $1.38_{-0.44}^{+0.51}$ & $1.59_{-0.68}^{+3.89}$ \\
\hline $0.25-0.50$ & $1.44_{-0.06}^{+0.04}$ & $4.6_{-0.9}^{+1.0}$ & $148.0 / 116$ & $1.34_{-0.03}^{+0.02}$ & $1.71_{-0.11}^{+0.22}$ & $1.39_{-0.12}^{+0.33}$ & $1.64_{-0.45}^{+0.52}$ \\
\hline $0.50-1.00$ & $1.51_{-0.02}^{+0.02}$ & $4.4_{-0.5}^{+0.4}$ & $201.5 / 116$ & $1.50_{-0.03}^{+0.03}$ & $1.76_{-0.05}^{+0.08}$ & $1.71_{-0.13}^{+0.29}$ & $1.87_{-0.22}^{+0.19}$ \\
\hline $1.00-2.00$ & $1.62_{-0.02}^{+0.01}$ & $2.7_{-0.5}^{+0.4}$ & $126.0 / 116$ & $1.63_{-0.02}^{+0.02}$ & $1.73_{-0.05}^{+0.03}$ & $1.95_{-0.24}^{+0.20}$ & $1.71_{-0.15}^{+0.17}$ \\
\hline $2.00-4.00$ & $2.04_{-0.01}^{+0.02}$ & $1.3_{-0.2}^{+0.2}$ & $213.4 / 116$ & $1.97_{-0.06}^{+0.04}$ & $2.11_{-0.04}^{+0.04}$ & $2.20_{-0.13}^{+0.43}$ & $2.19_{-0.10}^{+0.18}$ \\
\hline $4.00-8.00$ & $2.24_{-0.02}^{+0.02}$ & $1.6_{-0.2}^{+0.1}$ & $219.5 / 116$ & $2.10_{-0.04}^{+0.04}$ & $2.31_{-0.04}^{+0.04}$ & $2.29_{-0.23}^{+0.41}$ & $2.56_{-0.15}^{+0.14}$ \\
\hline
\end{tabular}

${ }^{a}$ Degrees of freedom.

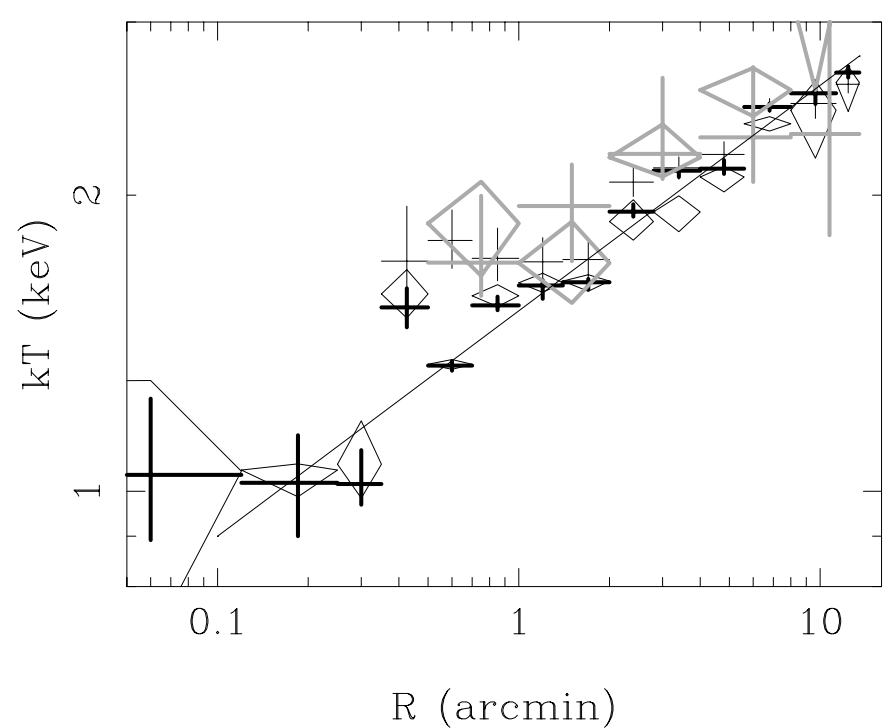

Fig. 15. Deprojected radial profile of the temperature obtained with EMOS, by fitting the spectra of whole energy band (bold black crosses), 0.7-1.3 keV (black diamonds), above $1.6 \mathrm{keV}$ (black crosses), 1.8-2.1 keV (gray crosses) and 2.3-2.7 keV (gray diamonds) with a APEC model. The solid line corresponds to the best fit regression line for the MEKAL model using the whole energy band of the EMOS.

Fe-K $\mathrm{K}_{\text {MEKAL }}, \mathrm{Fe}-\mathrm{L}_{\mathrm{MEKAL}}$, and Fe-L $\mathrm{L}_{\mathrm{APEC}}$ agree well each other. Within $R<1^{\prime}$, Fe-K MEKAL are consistent with Fe

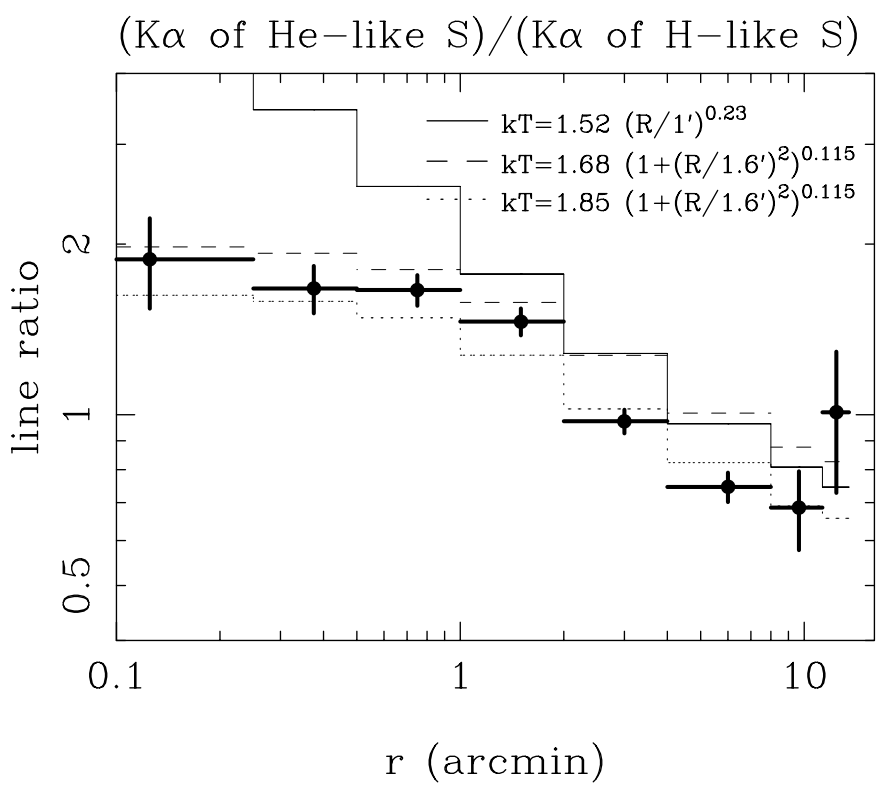

Fig. 16. Projected line ratios (EMOS) between K $\alpha$ of hydrogen and helium like S (closed circles). Errors correspond to $68 \%$ confidence level. Solid line and dashed lines represent the results from deprojected temperature profiles using APEC model, $k T_{\text {whole }}$ and $k T_{\text {hard }}$, respectively. We also plotted the profile for temperatures shifted by $10 \%$ (dotted line).

abundances obtained from the two temperature MEKAL model. However, Fe-K $\mathrm{K}_{\mathrm{APEC}}$ are systematically larger by 


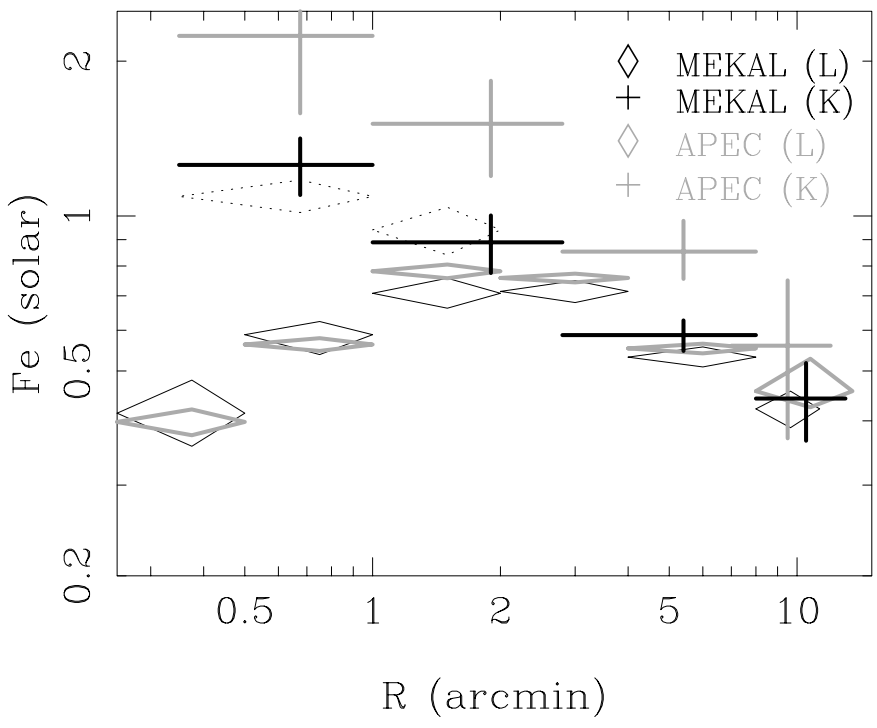

Fig. 17. Deprojected profile of Fe abundances obtained from the whole energy band (diamonds) and above $2 \mathrm{keV}$ (crosses), using the single temperature MEKAL model (black), APEC model (gray) and the two temperature MEKAL model (dotted).

$\sim 60 \%$. The strength of the projected Fe-K lines are almost a factor of 2 larger than the values calculated from the deprojected results (Fig. 18). In order to obtain the observed Fe-K line profile, the temperature should be shifted by $30 \%$ compared to the deprojected temperatures.

Adding another temperature component, the discrepancy of Fe-K lines become smaller (Fig. 14). However, the $\chi^{2}$ is still worse than the single temperature MEKAL model, and the radio of $\mathrm{S}$ lines cannot be well fitted.

In summary, the changes in the version 1.0 of the APEC code for the Ly $\alpha$ lines (H-like ions) are giving a much less consistent picture than the results from the MEKAL code. Therefore, we are not sure that the new atomic data included in APEC constitute an improvement.

\section{Intrinsic absorption}

The hydrogen column density, $N_{\mathrm{H}}$, derived from the single MEKAL fit is almost constant at $R>2^{\prime}$ (Table 2; Fig. 19). As summarized in Tables 2 and 5 , the $N_{\mathrm{H}}$ derived from the single APEC model is consistent with those from the single MEKAL model. The average value at $R>2^{\prime}$ is $1.5(1.2-1.7) \times 10^{20} \mathrm{~cm}^{-2}$ and $1.3(1.0-1.5) \times 10^{20} \mathrm{~cm}^{-2}$ for the EPN and EMOS, respectively. Within 2 arcmin, the two phase MEKAL model fit gives a consistent value with those at $R>2^{\prime}$, although the single MEKAL model fit gives a higher $N_{\mathrm{H}}$ value by several $\times 10^{20} \mathrm{~cm}^{-2}$. The response matrices of the EPN of September 2000 and November 2000 give systematically different values by $1 \sim 2 \times 10^{20} \mathrm{~cm}^{-2}$. Therefore, there may be a systematic uncertainty of a few $\times 10^{20} \mathrm{~cm}^{-2}$.

The value determined by the ROSAT PSPC observation is about $1.5(1.4-2.0) \times 10^{20} \mathrm{~cm}^{-2}$

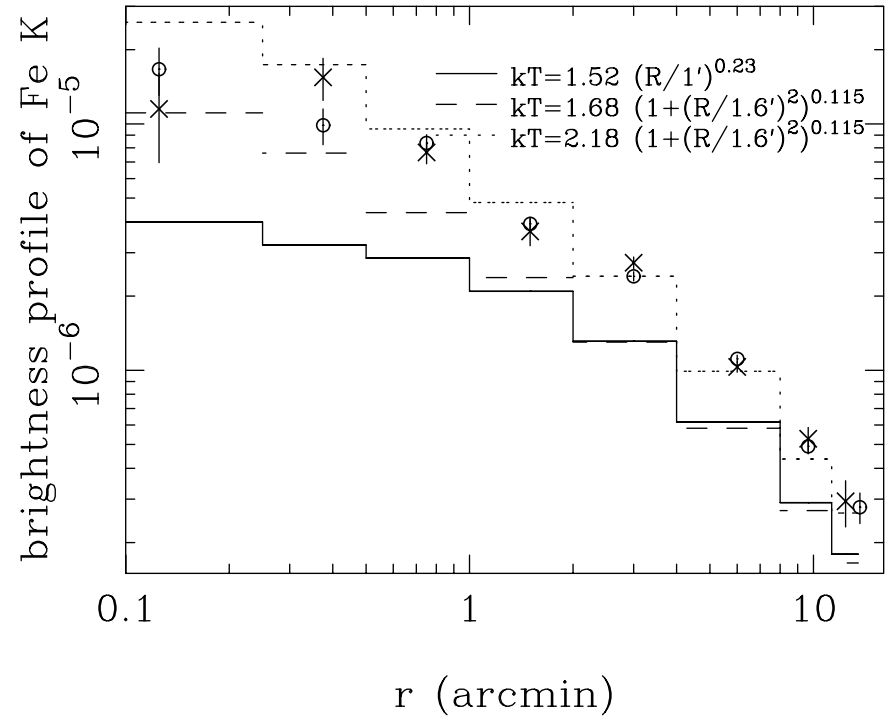

Fig. 18. Projected line brightness profile of Fe-K of the EMOS (open circles) and EPN (crosses). Errors correspond to 68\% confidence level. Solid line and dashed lines represent the results using APEC model and deprojected temperature profile, $k T_{\text {whole }}$ and $k T_{\text {hard }}$, respectively. We also plotted the profile for temperatures shifted by $30 \%$ (dotted line).

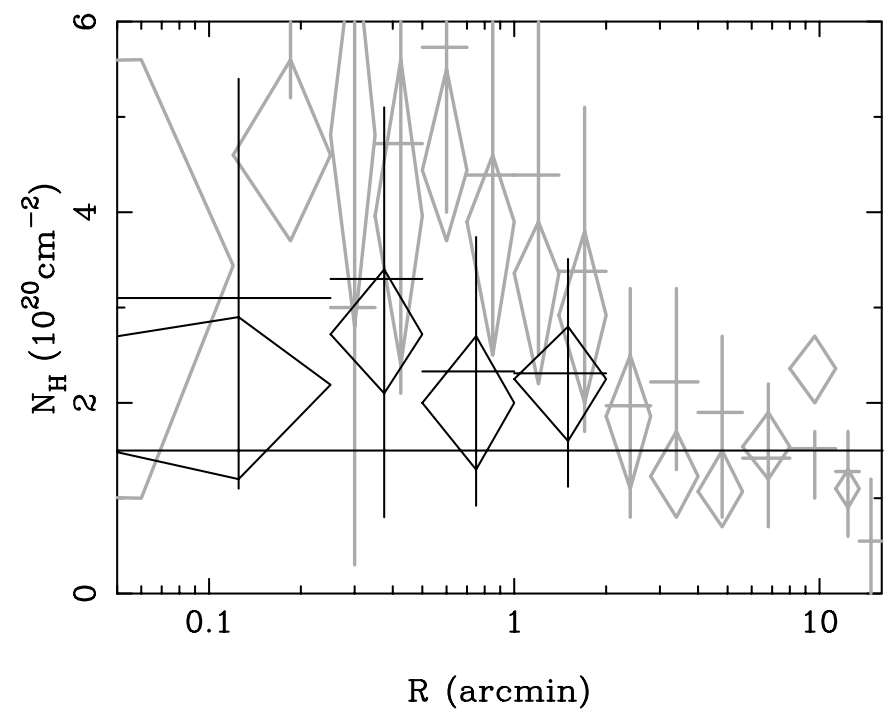

Fig. 19. The radial profile of hydrogen column density of EPN (crosses) and EMOS (diamonds) using the single temperature (gray) and the two temperature (black) MEKAL model fit. Holizontal line corresponds to the column density obtained from Nulsen \& Böhringer (1995).

(Nulsen \& Böhringer 1995; Böhringer 1999). The observed HI column density of our Galaxy by Stark et al. (1992) is $2.5 \times 10^{20} \mathrm{~cm}^{-2}$, which is slightly reduced to $1.8 \times 10^{20} \mathrm{~cm}^{-2}$, by Lieu et al. (1996). The observed $N_{\mathrm{H}}$ is consistent with the value obtained by ROSAT and also the Galactic column density. In addition, the power-law component from the central AGN shows no significant excess absorption. Thus, the intrinsic absorption should be negligible in the M 87 system. 
Table 6. Electron density profile.

\begin{tabular}{rcrc}
\hline$R$ & $\begin{array}{c}n_{\mathrm{e}} \\
\left(\mathrm{cm}^{-3}\right)\end{array}$ & $\begin{array}{r}R \\
(\operatorname{arcmin})\end{array}$ & $\begin{array}{c}n_{\mathrm{e}} \\
\left(\mathrm{cm}^{-3}\right)\end{array}$ \\
\hline $0.00-0.12$ & $1.2_{-1.1}^{+0.9} \times 10^{-1}$ & $2.00-2.80$ & $1.58_{-0.02}^{+0.02} \times 10^{-2}$ \\
$0.12-0.25$ & $1.2_{-0.2}^{+0.1} \times 10^{-1}$ & $2.80-4.00$ & $1.12_{-0.01}^{+0.01} \times 10^{-2}$ \\
$0.25-0.35$ & $9.2_{-0.7}^{+0.7} \times 10^{-2}$ & $4.00-5.60$ & $7.69_{-0.09}^{+0.09} \times 10^{-3}$ \\
$0.35-0.50$ & $7.2_{-0.4}^{+0.4} \times 10^{-2}$ & $5.60-8.00$ & $5.88_{-0.06}^{+0.05} \times 10^{-3}$ \\
$0.50-0.70$ & $5.6_{-0.3}^{+0.3} \times 10^{-2}$ & $8.00-11.3$ & $3.89_{-0.03}^{+0.02} \times 10^{-3}$ \\
$0.70-1.00$ & $3.9_{-0.5}^{+0.2} \times 10^{-2}$ & $11.30-13.5$ & $2.93_{-0.04}^{+0.04} \times 10^{-3}$ \\
$1.00-1.40$ & $2.8_{-0.1}^{+0.1} \times 10^{-2}$ & $13.50-16.0$ & $2.27_{-0.02}^{+0.02} \times 10^{-3}$ \\
$1.40-2.00$ & $1.9_{-0.1}^{+0.1} \times 10^{-2}$ & & \\
\hline
\end{tabular}

Table 7. result of double $\beta$ fit.

\begin{tabular}{rrr}
\hline $\begin{array}{r}R_{\text {core }} \\
(\operatorname{arcmin})\end{array}$ & $\beta$ & $\begin{array}{r}n_{\text {ecenter }} \\
\left(\mathrm{cm}^{-3}\right)\end{array}$ \\
\hline $0.35(0.27-0.44)$ & $0.42(0.39-0.46)$ & $0.13(0.11-0.16)$ \\
$4.4(4.2-4.7)$ & $0.47($ fix $)$ & $0.011(0.010-0.012)$ \\
\hline
\end{tabular}

\section{Density profile}

We have assumed $N_{\mathrm{H}}$ to be the average value of those obtained at $R>2^{\prime}$, since the higher $N_{\mathrm{H}}$ at $R<2^{\prime}$ from the single MEKAL model may be artificial. We have fixed the $\mathrm{C}, \mathrm{N}, \mathrm{O}$ abundance to $0.51\left(1+\left(R / 0.76^{\prime}\right)^{2}\right)^{-0.09}$, which is the best fit $\beta$-model of the abundance profile of these elements. Since the cooler component is distributed around the radio structures, and the hotter component is almost spherically symmetric, the latter should dominate. Thus, within 0.35 to $1.4^{\prime}$, we fitted the EPN spectra with a two component MEKAL model and adopted the electron density of the hotter component. The electron density $\left(n_{\mathrm{e}}\right)$, profile derived is shown in Table 6 and Fig. 20. These results are mostly consistent with those obtained by Nulsen \& Böhringer (1995), when considering the difference in the assumption of the abundance.

We fitted the $n_{\mathrm{e}}^{2}$ profile with a sum of two $\beta$ models, a compact and an extended one, both centered on M 87. We fixed the $\beta$ of the extended component to be 0.47 . which is the value obtained by ROSAT All Sky Survey (Böhringer et al. 1994). The obtained best-fit parameters are shown in Table 7 . The two $\beta$ components have distinct parameters, and cross over at $R \sim 2^{\prime}$, or $\sim 10 \mathrm{kpc}$.

\section{Dark matter profile}

The total mass $M(R)$ within a 3 -dimensional radius $R$, assuming hydrostatic equilibrium and spherical symmetry, is given by (e.g. Sarazin 1988),

$M(R)=-\frac{k T R}{G \mu m_{\mathrm{p}}}\left(\frac{\mathrm{d} \ln n}{\mathrm{~d} \ln R}+\frac{\mathrm{d} \ln T}{\mathrm{~d} \ln R}\right)$

where $m_{\mathrm{p}}$ is the proton mass, $k$ is the Boltzmann constant, $G$ is the constant of gravity, and $\mu \sim 0.63$ is the mean particle mass in units of $m_{\mathrm{p}}$.

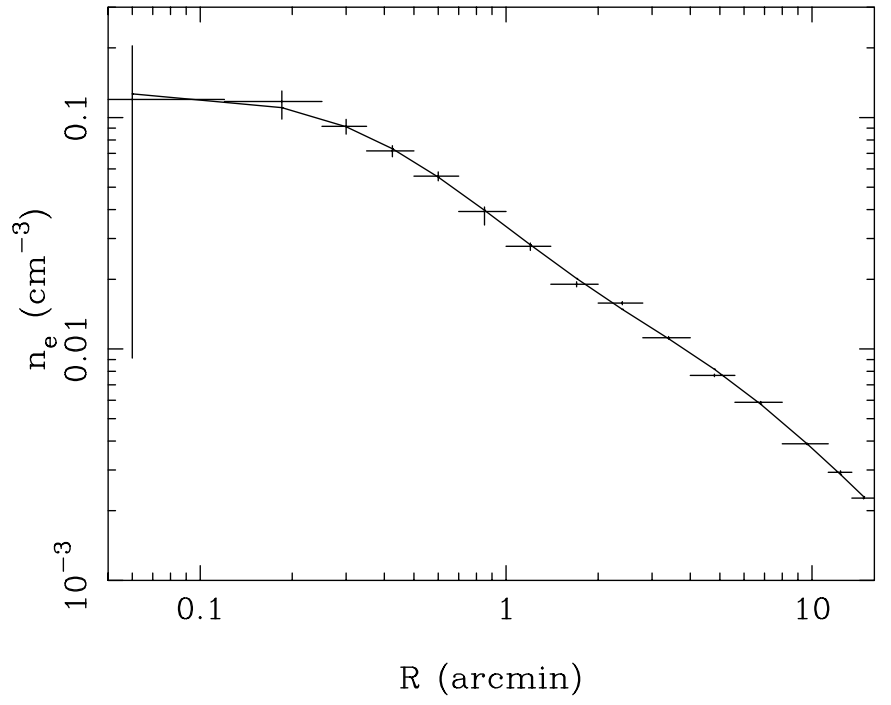

Fig. 20. Electron density profile. The solid line is the best-fit double- $\beta$ model.

Based on the deprojected temperature and density profile obtained in Sects. 3.3 and 5, we have calculated the gravitational mass of the M 87 system, together with the gas mass profile using the power-law relation in Fig. 3, and the double- $\beta$ relation approximated as in Table 7 . The upper and lower limit of the mass profile is calculated considering the uncertainty of the density gradient and the temperature. The temperature gradient is assumed to be smooth and expressed as the power-law relation. The upper and lower limit of the density gradient of the $i$ th shell is obtained from the ratio of the value within $i+1$ th shell to that within $i-1$ th shell. As plotted in Fig. 21, our result is mostly consistent with the dynamical mass profiles obtained by stars (Kronawitter et al. 2000) and globular clusters (Cohen \& Ryzhov 1997).

The calculated mass-to-blue light ratio (hereafter $\left.M / L_{\mathrm{B}}\right)$ of the stellar population ranges between 6 and 10 (e.g. Kronawitter et al. 2000). Assuming a constant stellar $M / L_{B}$ of 8 , in Fig. 21, we also plotted the stellar mass profile, calculated from the luminosity profile in Giraud (1999). Within nearly $0.5 r_{\mathrm{e}}$, the stellar velocity dispersion profile indicates that the dynamical $M / L_{B}$ is $\sim 11$ (e.g. Kronawitter et al. 2000). Here, $r_{\mathrm{e}}$ is the effective radius of M 87, which is $7.8 \mathrm{kpc}$ (de Vaucouleurs et al. 1991). Our mass profile indicates that at $R=1-2 r_{\mathrm{e}}$, the $M / L_{B}$ is about 17 and beyond this radius, the slope of the mass profile increases and $M / L_{B}$ reaches $\sim 200$ at $10 r_{\mathrm{e}}$.

\section{Application of the cooling flow model}

\subsection{Mass flow profile from imaging data}

The steady state cooling flow structure in a spherically symmetric cluster is obtained by,

$$
\begin{aligned}
\frac{1}{R^{2}} \frac{\mathrm{d}}{\mathrm{d} R}\left\{R^{2} \rho_{\mathrm{g}} v\left\{\frac{v^{2}}{2}+\frac{5}{2} \frac{P}{\rho_{\mathrm{g}}}+\phi(R)\right\}\right\} & =n_{\mathrm{e}}^{2} \Lambda \\
\dot{M} & =4 \pi R^{2} \rho_{\mathrm{g}} v
\end{aligned}
$$




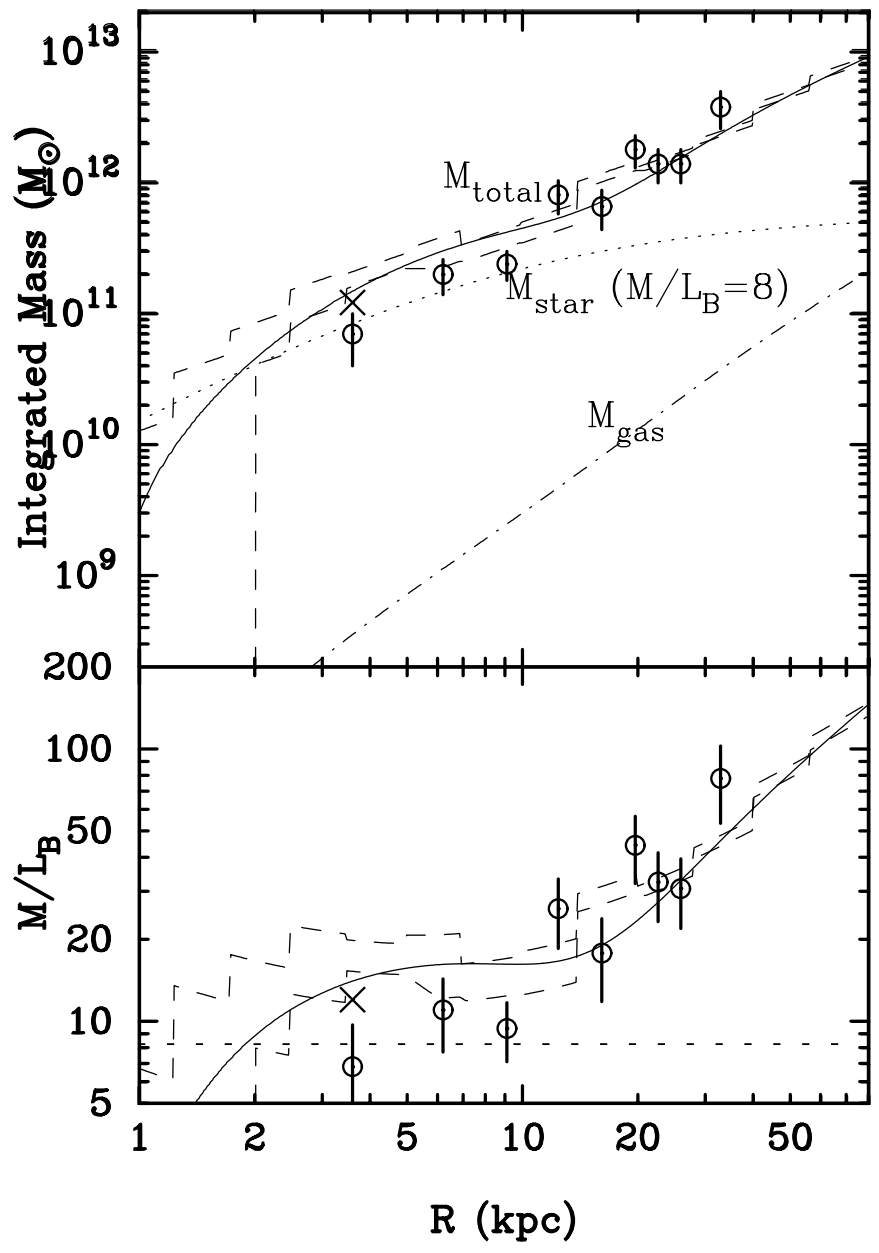

Fig. 21. (Upper panel) integrated mass profiles of the total gravitating matter (dashed lines for upper and lower limit and solid line for the double $\beta$ model fit), the X-ray emitting plasma (dot-dashed line), and the stellar component (dotted line) assuming stellar $M / L_{B}$ to be 8 , in the M 87 system. The dynamical mass obtained by stars (cross; Kronawitter et al. 2000) and globular clusters (open circles; Cohen \& Ryzhov 1997) are also shown. (Lower panel) Total mass-to-light ratio $\left(M / L_{B}\right)$ of the system. The meanings of symbols and lines are the same as in the upper panel.

(e.g. Sarazin 1988), where $\rho_{\mathrm{g}}, v$, and $P$ are, respectively, the gas density, velocity, and pressure, and $\phi(R)$ is the gravitational potential, $\Lambda$ is the emissivity.

Even within the optical radii of elliptical galaxies, the $\mathrm{X}$-ray luminosity is a factor of 20 larger than heating by stellar mass loss (Matsushita 2001). Therefore, we can neglect this component as a sufficient heat source to balance cooling. The cooling time is lower than the Hubble time within $R \sim 50 \mathrm{kpc}$ (Fig. 22). As shown in Fig. 22, the $\dot{M}_{\text {I }}$ obtained is roughly proportional to the radius. At $R \sim 50 \mathrm{kpc}, \dot{M}_{\mathrm{I}}$ is $10 M_{\odot}$, which is consistent to the classical value obtained by Stewart et al. (1984) from the Einstein observation.

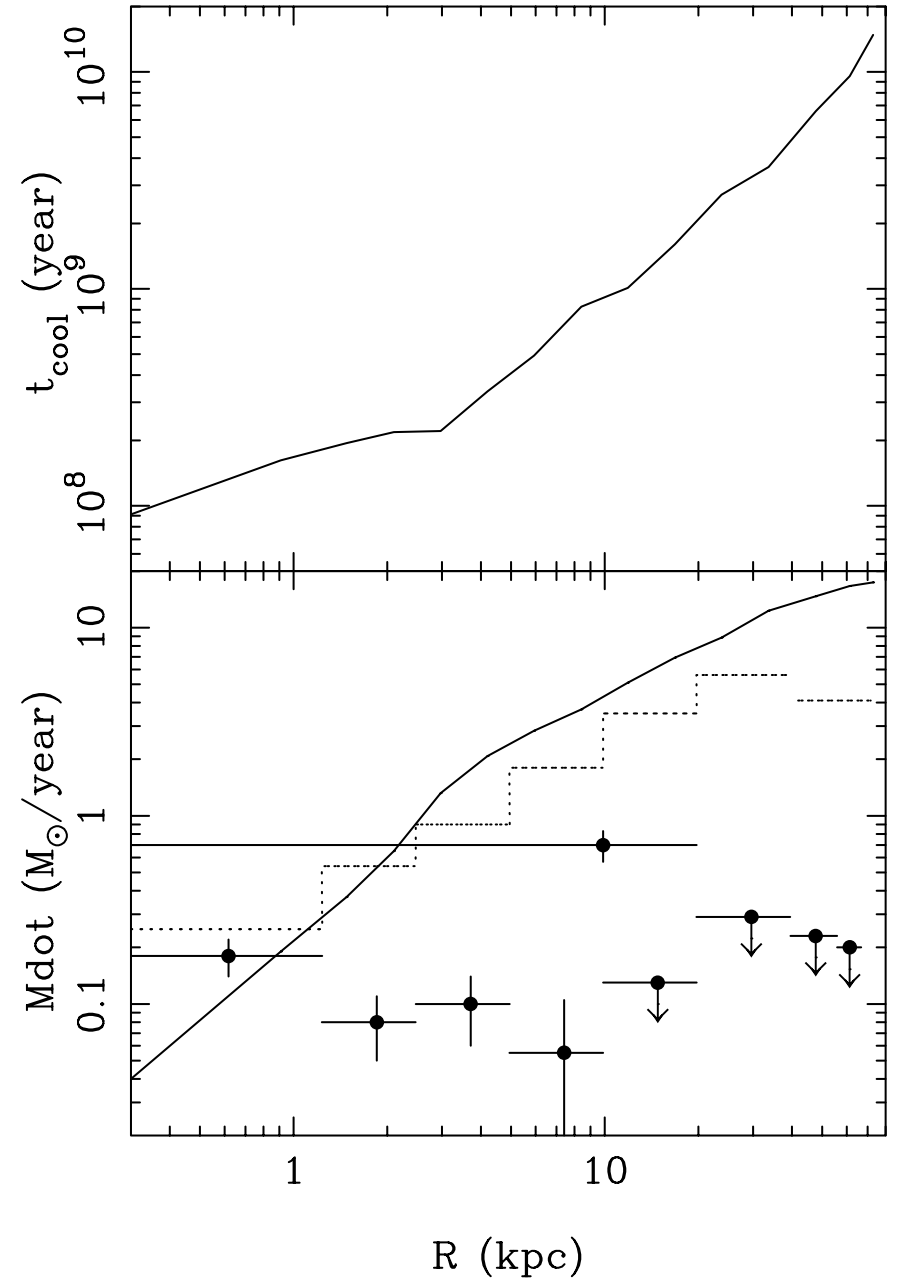

Fig. 22. Cooling time (upper panel) and $\dot{M}_{\mathrm{I}}$ profile (lower panel). Data corresponds to $\dot{M}_{\mathrm{S}}$, assuming cooling to $0 \mathrm{keV}$. The dotted line is the differential of the $\dot{M}_{\mathrm{I}}$, the mass deposition rate in each shell.

\subsection{Spectral fitting using a spectral cooling flow model}

With the RGS we can observe only the very central core of cooling flow clusters. In contrast, using the EPN, we can accumulate spectra within the entire cooling flow region.

At $R=4^{\prime}$, the derived cooling time is only a few Gyr and $\dot{M}_{\mathrm{I}}$ is $8 M_{\odot} \mathrm{y}^{-1}$, respectively. The net effect of resonance scattering is negligible for the radiative output in this integrated circular region. Without any heating, the gas should cool down to $0 \mathrm{keV}$, emitting X-rays at intermediate temperatures. Therefore, the spectral cooling rate, $\dot{M}_{\mathrm{S}}$, within the radius should be $8 M_{\odot} \mathrm{y}^{-1}$.

We fitted the deprojected spectra within $R<4^{\prime}$ with a cooling flow model using the MEKAL code modified by photoelectric absorption. As in other cooling flow clusters observed by RGS, in order to fit the spectra, we still need a cut-off temperature, low $T$, which is found to be $1.4 \mathrm{keV}$ (Table 8). Figure 23 shows the EPN spectrum within $R<4^{\prime}$, fitted with the cooling flow model. We also plotted in the figure, another cooling flow model with low $T=0.1 \mathrm{keV}$, fitted only to the data in the spectral 
Table 8. Result of spectral fitting using a spectral cooling flow model.

\begin{tabular}{|c|c|c|c|c|c|c|c|c|}
\hline $\begin{array}{r}R \\
\operatorname{arcmin}\end{array}$ & model & $\begin{array}{c}k T_{\text {vmekal }} \\
(\mathrm{keV})\end{array}$ & $\begin{array}{c}\operatorname{low} k T_{\mathrm{cf}} \\
(\mathrm{keV})\end{array}$ & $\begin{array}{c}\operatorname{high} k T_{\mathrm{cf}} \\
(\mathrm{keV})\end{array}$ & $\begin{array}{c}N_{\mathrm{H}_{\mathrm{MEKAL}}} \\
\left(10^{20} \mathrm{~cm}^{-2}\right)\end{array}$ & $\begin{array}{c}N_{\mathrm{H}_{\mathrm{MEKAL}}} \\
\left(10^{20} \mathrm{~cm}^{-2}\right)\end{array}$ & $\begin{array}{r}\dot{M} \\
\left(M_{\odot}\right)\end{array}$ & $\chi^{2} / \mu$ \\
\hline $0.0-4.0$ & mkcflow & - & $1.442_{-0.002}^{+0.002}$ & $2.30_{-0.02}^{+0.02}$ & $1.3_{-0.2}^{+0.2}$ & $=N_{\mathrm{H}_{\mathrm{MEKAL}}}$ & $13.8_{-0.01}^{+0.01}$ & $370 / 191$ \\
\hline $0.0-4.0$ & mekal+mkcflow & $1.75_{-0.01}^{+0.01}$ & 0.10 (fix) & $=k T_{\mathrm{vmekal}}$ & $2.9_{-0.2}^{+0.2}$ & $=N_{\mathrm{H}_{\mathrm{MEKAL}}}$ & $0.63_{-0.08}^{+0.20}$ & $416 / 130$ \\
\hline $0.0-4.0$ & mekal+mkcflow & 1.58 & 0.10 (fix) & $=k T_{\mathrm{vmekal}}$ & 1.5 & 117 & 8.0 (fix) & $1088 / 192$ \\
\hline $0.0-0.25$ & mekal+mkcflow & $1.7_{-0.6}^{+0.8}$ & 0.10 (fix) & $=k T_{\mathrm{vmekal}}$ & 1.5 (fix) & 1.5 (fix) & $0.18_{-0.04}^{+0.03}$ & $30 / 51$ \\
\hline $0.25-0.5$ & mekal+mkcflow & $1.58_{-0.08}^{+0.08}$ & 0.10 (fix) & $=k T_{\mathrm{vmekal}}$ & $2.5_{-0.5}^{+0.6}$ & $=N_{\mathrm{H}_{\mathrm{MEKAL}}}$ & $0.08_{-0.02}^{+0.03}$ & $62 / 51$ \\
\hline $0.5-1.0$ & mekal+mkcflow & $1.52_{-0.06}^{+0.05}$ & 0.10 (fix) & $=k T_{\mathrm{vmekal}}$ & $4.4_{-1.0}^{+1.0}$ & $=N_{\mathrm{H}_{\mathrm{MEKAL}}}$ & $0.10_{-0.03}^{+0.04}$ & $91 / 51$ \\
\hline $1.0-2.0$ & mekal+mkcflow & $1.65_{-0.06}^{+0.06}$ & 0.10 (fix) & $=k T_{\mathrm{vmekal}}$ & $2.8_{-1.3}^{+1.3}$ & $=N_{\mathrm{H}_{\mathrm{MEKAL}}}$ & $0.05_{-0.04}^{+0.05}$ & $78 / 51$ \\
\hline $2.0-4.0$ & mekal+mkcflow & $1.97_{-0.05}^{+0.05}$ & 0.10 (fix) & $=k T_{\mathrm{vmekal}}$ & $1.5_{-0.5}^{+0.5}$ & $=N_{\mathrm{H}_{\mathrm{MEKAL}}}$ & $<0.13$ & $103 / 51$ \\
\hline $4.0-8.0$ & mekal + mkcflow & $2.30_{-0.08}^{+0.07}$ & 0.10 (fix) & $=k T_{\mathrm{vmekal}}$ & $1.6_{-0.4}^{+0.4}$ & $=N_{\mathrm{H}_{\mathrm{MEKAL}}}$ & $<0.29$ & $58 / 51$ \\
\hline $8.0-11.3$ & mekal + mkcflow & $2.60_{-0.13}^{+0.12}$ & 0.10 (fix) & $=k T_{\mathrm{vmekal}}$ & $1.8_{-0.9}^{+0.8}$ & $=N_{\mathrm{H}_{\mathrm{MEKAL}}}$ & $<0.23$ & $51 / 51$ \\
\hline $11.3-13.5$ & mekal + mkcflow & $2.66_{-0.16}^{+0.16}$ & 0.10 (fix) & $=k T_{\mathrm{vmekal}}$ & $1.5_{-0.6}^{+0.6}$ & $=N_{\mathrm{H}_{\mathrm{MEKAL}}}$ & $<0.20$ & $111 / 51$ \\
\hline
\end{tabular}

range above $1.2 \mathrm{keV}$. The difference in the continuum level between 0.2 and $0.5 \mathrm{keV}$ is due to the instrumental lowenergy tail of Fe-L and O-K lines. The component cooling radiatively to $0 \mathrm{keV}$ should emit strong $\mathrm{Fe}-\mathrm{L}$ lines between 0.6 to $1.0 \mathrm{keV}$, which are not seen in the spectrum.

When adding an isothermal MEKAL component, we can fit the spectra with a cooling flow component with low $T=0.1 \mathrm{keV}$. However, $\dot{M}_{\mathrm{S}}$ is only $0.8 M_{\odot} \mathrm{y}^{-1}$, which is a factor of 10 smaller than $\dot{M}_{\text {I }}$ at the same radius.

\subsubsection{Test of a bimodal abundance model}

Fabian et al. (2001) made a proposal to solve the cooling flow problem by a scenario in which metals in the ICM are not uniformly distributed. In this suggestion, a metal poor part of the gas cools without emitting lines and a metal rich part cools rapidly. In this way, the total strength of Fe-L line emission should be reduced. A bimodal abundance model, with 0.01 solar abundances and 10 times higher abundances than the single abundance model and mass deposition rates of 7.3 and $0.7 M_{\odot} / \mathrm{yr}$, respectively, is shown in Fig. 23. It is very different from the observed spectrum in the Fe-L structure and strength of K- $\alpha$ lines (Fig. 23), since it reduces not only Fe-L lines but also K lines of Si, S, Ar, Ca, and Fe (see also Böhringer et al. 2002). In order to obtain the observed strength of $\mathrm{K}$ lines with these mass deposition rates, the abundances in the high metallicity component should be $\sim 100$ solar. Therefore, we have tried another bimodal metal abundance model, where the mass deposition rates of the two components are allowed to be free parameters, and fitted the spectra above $1 \mathrm{keV}$. Figure 23 also shows the best fit model, extrapolated to lower energies. With the mass deposition rates of 4 and $4 M_{\odot} / \mathrm{yr}$, for the metal rich and metal poor components respectively, the spectra above $\sim 1 \mathrm{keV}$ can be fitted. Because of the dependence of the cooling function on metallicity, the Fe-L strength below $0.9 \mathrm{keV}$ is slightly reduced compared to that of the single abundance model. However, the model shows very strong Fe-L lines below $0.9 \mathrm{keV}$. We then added absorption for the metal rich components and then allowed the abundances of the metal rich component to vary. Although including a column density of $5 \times 10^{21} \mathrm{~cm}^{-2}$ dramatically improved the fit, the fit is much worse compared to the fit with the cut-off temperature. In addition, such high absorption heavily reduces the spectra around the $0.67 \mathrm{keV}$ $\mathrm{O}$ line. Increasing the $\mathrm{O}$ abundance of the metal rich components does not solve the problem, since it reduces the strength of Fe-L lines due to the difference in the cooling function. Therefore, there must exist an unabsorbed component whose $\mathrm{O}$ abundance is not so peculiar. For intrinsic absorption, more details are discussed also on Böhringer et al. (2002).

In summary, any multi-abundance model cannot explain the observed Fe-L profiles. In order to explain it, we need a sharp cut off in the temperature distribution.

\subsubsection{Radial profile of spectral cooling flow component}

The observed radial profile of $\dot{M}_{\mathrm{I}}$ is not constant, but roughly proportional to $R$. The differential of $\dot{M}_{\text {I }}$ between a shell and next shell implies that we should observe a cooling flow component in the spectrum of each radial shell. We thus fitted deprojected spectra with a cooling flow model with low $T=0.1 \mathrm{keV}$, and an isothermal MEKAL model. The result is summarized in Table 8 and Fig. 22. Within $R<1^{\prime}$, the fit has improved from the single temperature fit. However, the $\dot{M}_{\mathrm{S}}$ values, are a factor of 10 to 20 lower than the differential of $\dot{M}_{\mathrm{I}}$. Only for the innermost shell, $\dot{M}_{\mathrm{S}}$ and $\dot{M}_{\mathrm{I}}$ are consistent with each other. 

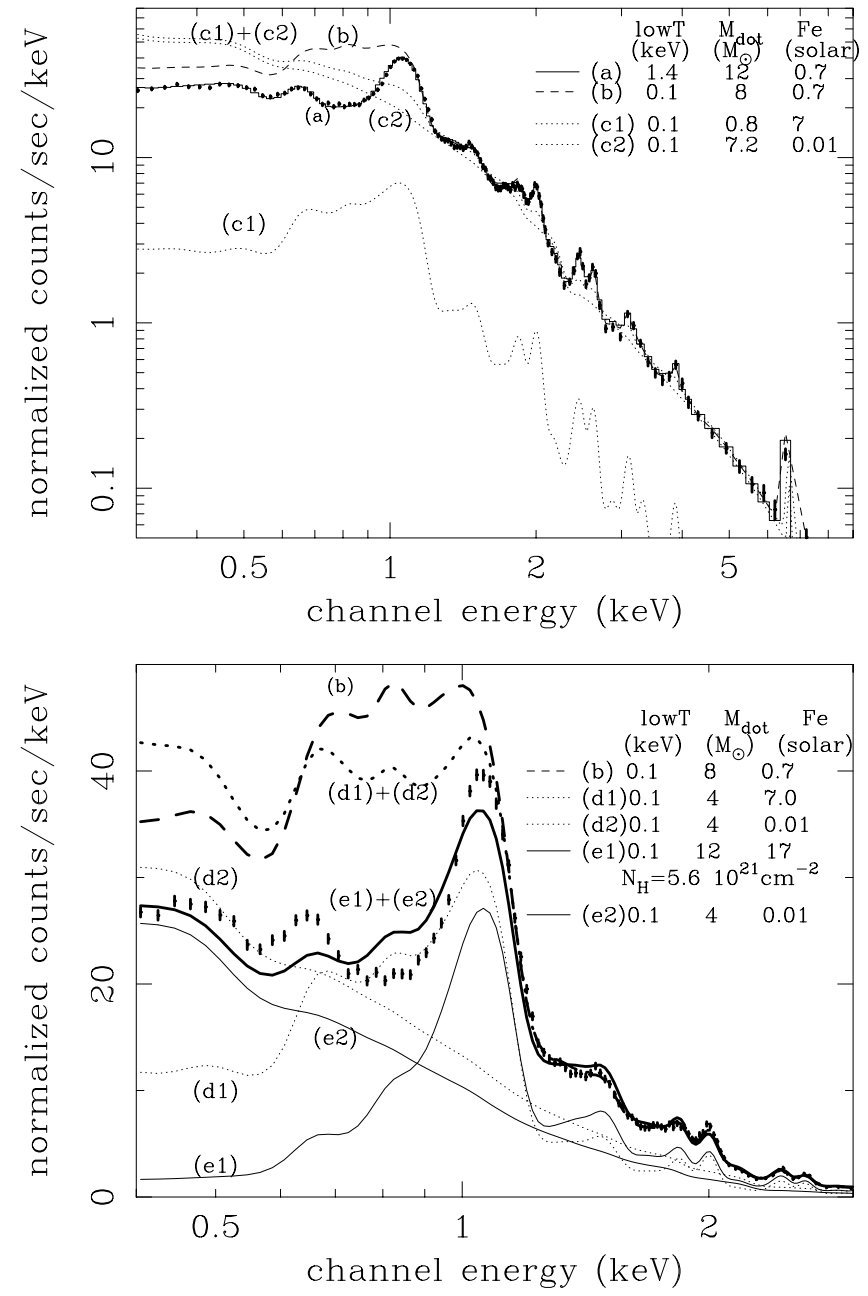

Fig. 23. Deprojected spectra within $R<4^{\prime}$ fitted with a cooling flow model with a cut-off temperature, low $T=1.44 \mathrm{keV}$ (solid line, a). The bold dotted line (b) shows the model with cooling to $0.1 \mathrm{keV}$. The thin dashed lines of the upper panel, (c1) and (c2), corresponds to the model with a model with 10 times higher metallicity and a model with low metal abundance, respectively. The spectral cooling flow rates are summarized in the panel. The sum of (c1) and (c2) is also plotted. The dotted lines of the lower panel, (d1) and (d2) are components of another bimodal metal abundance mdoel, and the solid lines of (e1) and (e2) corresponds to the model with excess absorption applied for the metal rich components.

\section{Discussion}

\subsection{Temperature structure}

The deprojected spectra of M 87 strongly indicate that excluding the region associated to the radio structures, the ICM is single phase; at a given radius, the ICM is dominated by one temperature component. We cannot detect any multi-phase cooling flow component as seen by Allen et al. (2001). The ICM is also not characterized by a twophase structure as suggested by ASCA observations of the Centaurus cluster (Fukazawa et al. 1994; Ikebe et al. 1999), and M 87 (Matsumoto et al. 1996).

When excluding the regions with the radio structures, the ICM is nearly spherically symmetric. Using the
MEKAL model, a single phase model can fit the deprojected spectra well at $R>2^{\prime}$. The temperature profile has a positive temperature gradient, $\sim 1.7 \mathrm{keV}$ at $R=1^{\prime}$ and $\sim 2.5 \mathrm{keV}$ at $R=15^{\prime}$. The consistency of the temperatures obtained by the continuum, Fe-L region, Si, and $\mathrm{S}$ line ratios, Fe-L to Fe-K ratio indicate that the ICM is dominated by a single temperature component. The regions associated to the radio structures have an additional temperature components of $1 \mathrm{keV}$. At $R<2^{\prime}$, since it is difficult to filter the complicated radio structures within $2^{\prime}$ with the XMM spatial resolution, we observe the major ICM components with the temperature of $1.7 \mathrm{keV}$, and small amount of the $1 \mathrm{keV}$ components. The spatial distribution of the latter component indicates that the cooler component also relates to the region corresponding to the radio lobes (see also Belsole et al. 2001).

The R-S model gives a temperature lower by $20 \%$ than MEKAL model. In order to fit the spectra, the R$\mathrm{S}$ model requires an additional high temperature component. However, the two temperature R-S model cannot explain the observed $\mathrm{Si}$ and $\mathrm{S}$ line ratios.

Except for the Fe-K line strength, the APEC temperatures are consistent with the MEKAL models within $10-15 \%$. Due to changes of the strength of $\mathrm{K} \alpha$ lines, the single phase APEC model cannot explain the strength of Fe-K lines, although it can fit the Fe-L and continuum simultaneously. The $\mathrm{S}$ line ratio is discrepancy by a factor of 1.5 , but it introduces only a $10 \%$ difference in the temperature structure.

Resonant scattering of line emission should be important in dense core of galaxy clusters. (Gil'fanov et al. 1987; Tawara et al. 1997). Shigeyama (1998) calculated the effect on M 87 system, and found that surface brightness profile considerably decreased within $1-2^{\prime}$ because many Fe-L lines are optically thick. In M 87, Böhringer et al. (2001) suggested that the resonant scattering may explain the observed sharp abundance drop at center. This effect may also change the spectra and the Fe-L line profile in the central region. Part of the $1 \mathrm{keV}$ component may be due to this effect. Therefore, in order to study the temperature structure within $1-2^{\prime}$ arcmin exactly, we have to consider effect of the resonance scattering. However, since the temperature obtained from the continuum spectra is close to that from the whole energy band, the main temperature component should be similar to the obtained values. At least using the MEKAL model, the strength of the Fe-L and $\mathrm{Fe}-\mathrm{K}$ are consistent with each other, which have a different dependence on the resonance line scattering. In addition, most of the $1 \mathrm{keV}$ component should be related to the radio activity which has a complicated spatial distribution (Belsole et al. 2001). Most of the resonantly scattered light will be reemitted at slightly larger projected radii. Therefore integrating over the region, $R<4^{\prime}$ will reduce the net effect of scattering and the spectra will hardly be disturbed.

Matsushita (2001) found that normal elliptical galaxies have $\beta_{\text {spec }}=1$. The central stellar velocity dispersion of $\mathrm{M} 87$ is $350 \mathrm{~km} \mathrm{~s}^{-1}$ (Whitmore et al. 1985). 
The assumption of $\beta_{\text {spec }}=1$ implies a temperature of $0.8 \mathrm{keV}$, which agrees well with the observed central ICM temperature of $M 87$. The ICM temperature at $10-15^{\prime}$ of $2.5 \mathrm{keV}$ also agrees with the velocity dispersion of earlytype galaxies of the Virgo cluster, which is $570 \mathrm{~km} \mathrm{~s}^{-1}$ (Binggeli \& Tammann 1987). The observed constancy in the temperature suggests that the central cool temperature mainly reflects the galaxy potential, even though some part of the cool component may be produced by a small cooling flow in the central region.

\subsection{Intrinsic absorption}

The hydrogen column density is almost flat within the field of view. In addition, the spectrum of the central AGN does not show any absorption feature. Therefore, intrinsic absorption should be negligible in the M 87 system. If there is excess $N_{\mathrm{H}}$ of the order of $10^{20} \mathrm{~cm}^{-2}$ and the absorbing matter is uniformly distributed within $10 \mathrm{kpc}$ and $50 \mathrm{kpc}$, the mass of the absorbing matters is $10^{8} M_{\odot}$ and $10^{9} M_{\odot}$, respectively, which is much smaller than the cooling flow mass deposition rate accumulated over a Hubble time.

\subsection{Potential structure of ellipticals and clusters of galaxies}

We have determined the gravitational mass profile of the M 87 system, directly from the observed density and temperature profiles. At $0.5-2 r_{\mathrm{e}}, M / L_{B}$ of the system is constant at $\sim 17$. Beyond this radius, it starts to increase to $\sim 200$ at $80 \mathrm{kpc}$.

This structure is essentially the same feature as seen in the Fornax cluster (Ikebe et al. 1996), the Centaurus cluster (Ikebe et al. 1999) and NGC 4636 (Matsushita et al. 1998). Figure 24 summarizes the gravitational mass profiles of the $3 \mathrm{cD}$ galaxies and NGC 4636. These galaxies have similar hierarchical mass profiles; the gravitational mass gradually increases up to several $r_{\mathrm{e}}$, where the gravitational to stellar mass ratio is common among galaxies. Then, $M / L$ start to increase steeply.

Before the total to stellar mass ratio increases steeply, the total $M / L_{B}$ is $\sim 17$ at $1 r_{\mathrm{e}}$, and $\sim 30$ even at $10 r_{\mathrm{e}}$. This feature should reflect the potential of elliptical galaxies. The $M / L_{B}$ of the central core of these galaxies obtained by the dynamics and the same ratio obtained by the stellar population synthesis models are consistent with each other giving a typical value of 6-10 (e.g. Kronawitter et al. 2000; Gerhard et al. 2001). Considering the observed metallicity gradient of stars, the stellar $M / L_{B}$ should be slightly smaller in the outer regions (e.g. Kodama \& Arimoto 1997). Our result suggests that the dark mass distribution of elliptical galaxies is slightly more extended than those of stars. It is thought that elliptical galaxies have a 10 times larger amount of dark mass than stellar mass (e.g. Ciotti et al. 1991). However, the galaxies themselves may not contain such large amount of mass. This result should be important for the study of the origin of the dark

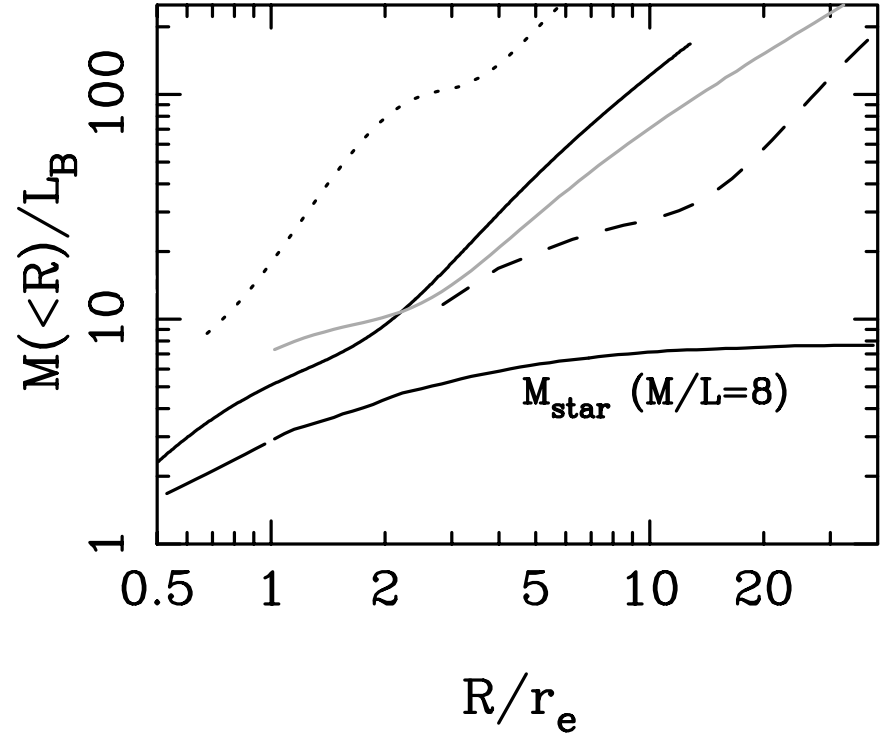

Fig. 24. Integrated mass profiles of the total gravitating matter scaled by $L_{B}$, and the stellar component (solid line) in the M 87 (solid line; $L_{\mathrm{X}} / L_{\sigma}=22$ ), the Centaurus cluster (dotted line; Ikebe et al. 1999; $L_{\mathrm{X}} / L_{\sigma}=47$ ), NGC 1399 (dashed line; Ikebe et al. 1996; $L_{\mathrm{X}} / L_{\sigma}=3$ ), and NGC 4636 (gray line; Matsushita et al. 1998; $L_{\mathrm{X}} / L_{\sigma}=12$ ). $L_{\mathrm{X}}$ and $L_{\sigma}$ means the ISM luminosity within $4 r_{\mathrm{e}}$ and kinematical energy input from stellar mass loss (Matsushita 2001).

matter content in elliptical galaxies and also for the study of the formation and evolution of these galaxies.

The scatter of the X-ray to blue luminosity ratio of early-type galaxies has long been a problem (e.g. Canizares et al. 1987). Matsushita (2001) found that $L_{\mathrm{X}}$ of most of the early-type galaxies is well explained by a kinematical heating of the gas supplied by stellar mass loss $\left(L_{\sigma}\right)$. All of the galaxies which have much larger $L_{\mathrm{X}}$ than $L_{\sigma}$ have very extended X-ray emission. Figure 24 shows that the galaxies with a larger $L_{\mathrm{X}} / L_{\sigma}$ have smaller radii of the starting point of the strong increase of $M / L$, when scaled $r_{\mathrm{e}}$. In other words, the X-ray luminosity of $\mathrm{cD}$ galaxies may be determined in relation to their potential structure, as indicated by Matsushita (2001).

\subsection{Problem of standard cooling flow model}

The standard cooling flow model indicates that the ICM is multi-temperature on small scales, since it is thought that mass is deposited within the whole cooling flow region. The cooling matter should emit strong Fe-L lines below $0.9 \mathrm{keV}$. We cannot detect a significant spectral cooling flow component from the whole field of view of the detector and the ICM at any given radius is dominated by a single temperature component. The upper limit on $\dot{M}_{\mathrm{S}}$ is an order of magnitude smaller than $\dot{M}_{\mathrm{I}}$. A bimodal metal abundance distribution model cannot explain the observed Fe-L profile.

The resonance scattering may reduce the strength of some resonance lines. However, all the Fe-L lines below 
$0.9 \mathrm{keV}$, which includes both resonance lines and nonresonance lines, are suppressed compared with the expected value for a cooling flow. In addition, the spectral cooling flow component is also small where the resonance scattering is not effective. Therefore, the resonant scattering can not be responsible for the suppresion of the Fe-L lines below $0.9 \mathrm{keV}$.

At $1 r_{\mathrm{e}}\left(1.6^{\prime}\right)$ of $\mathrm{M} 87, \dot{M}_{\mathrm{I}}$ is $\sim 4 M_{\odot} \mathrm{yr}^{-1}$. In contrast, the upper limit on $\dot{M}_{\mathrm{S}}$ is $\sim 0.4 M_{\odot} \mathrm{yr}^{-1}$. This value is close to the stellar mass loss rate within $1 r_{\mathrm{e}}$, which is $0.5 M_{\odot} \mathrm{yr}^{-1}$. At this radius, $t_{\text {cool }}$ is $10^{9} \mathrm{yr}$ and the gas mass is $2 \times 10^{9} M_{\odot}$. Therefore, considering the observed abundance gradient, the standard cooling flow model indicate that the gas should be diluted by metal poor surrounding gas. However, the observed Fe abundance of M 87 within $1 r_{\mathrm{e}}, \sim 1$ solar, is similar to those of normal X-ray luminous galaxies, which are also about $\sim 1$ solar (Matsushita et al. 1997, 2000). Note that the observed ISM abundance of NGC 4636 from the RGS spectra is 0.9 solar (Xu et al. 2001). In these systems, the enrichment has to be compared with a mass flow rate of $\sim 1 M_{\odot} \mathrm{yr}^{-1}$ as implied by the standard cooling flow models for these galaxies. For the case of NGC 4636, the mass flow rate is determined to be $\sim 1 M_{\odot} \mathrm{yr}^{-1}$ (Bregman et al. 2001). The effect of the dilution by ICM should be determined by a ratio of the mass flow rate and the optical luminosity since metals ejected from a galaxy should be proportional to the stellar luminosity. Within $1 r_{\mathrm{e}}$, the former rate of NGC 4636 is a factor of 10 smaller than that of M 87, while the latter is only a factor of 2.5 smaller. In a much larger cooling flow as exists in M 87 in which the Fe enrichment is supplied by a stellar population comparable to that of normal ellipticals, the relative enrichment will be less and the abundance gradient must be reduced in contrast to the observed data. Therefore, the overall cooling flow expected from the classical galaxy cluster cooling flow model should not exist in M 87, although a smallscale cooling flow, with a mass deposition rate close to the stellar mass loss rate, may exists in the central core of the classical cooling flow regions.

\section{Conclusion}

The major result of this paper is strong evidence that the ICM in the halo of M 87 is isothermal locally, except probably for the very central region, where more than one temperature component is present. This could be partly due to the interaction effects of the ICM with the jet and the radio lobes of $\mathrm{M} 87$. This finding and the fact that no spectral signature of low temperature components below temperatures of $0.8 \mathrm{keV}$ are observed is in disagreement with the standard cooling flow model which predicts a strongly multi-phase structure of the ICM and a distinct temperature distribution and resulting spectrum with a clear signature of low temperature components. We have also shown that strong inhomogeneities in the metal distribution cannot resolve this problem. Therefore we conclude that the scenario for the dense gas with short cooling time in the centers of clusters needs to be revised. Probably heating processes that can substantially reduce the mass deposition have to be reconsidered, like the energy input of the central AGN. The central temperature closely reflects the gravitational potential depth of the central galaxy, rather than the existence of a cooling flow.

Acknowledgements. This work was supported by the Japan Society for the Promotion of Science (JSPS) through its Postdoctoral Fellowship for Research Abroad and Research Fellowships for Young Scientists.

\section{References}

Allen, S. W., \& Fabian, A. C.,1994, MNRAS, 269, 409

Allen, S. W. 2000, MNRAS, 315, 269

Allen, S. W., Fabian, A. C., Johnstone, R. M., Arnaud, K., \& Nulsen, P. H. E. 2001, MNRAS, 322, 589

Anders, E., \& Grevesse, N. 1989, Geochimica et Cosmochimica Acta, 53, 197

Arnaud, M., \& Rothenflug, M. 1985, A\&AS, 60, 425

Arnaud, M., \& Raymond, J. 1992, A\&A, 398, 394

Belsole, E., Sauvageot, J. L., Böhringer, H., et al. 2001, A\&A, 365, L188

Binggeli, B., \& Tammann, G. A. 1987, AJ, 94, 251

Böhringer, H., Briel, U. G., Schwarz, R. A., et al. 1994, Nature, 368,828

Böhringer, H., Nulsen, P. E. J., Braun, R., \& Fabian, A. C. 1995, MNRAS, 274, 67

Böhringer, H. 1999, in Diffuse Thermal and Relativistic Plasma in Galaxy Clusters, ed. H. Böhringer, L. Feretti, \& P. Schuecker, MPE Report, 115

Böhringer, H., Belsole, E., Kennea, J., et al. 2001, A\&A, 365, L181

Böhringer, H., Matsushita, K., Churazov, E., Ikebe, Y., \& Chen, Y. 2002, A\&A, 382, 804

Bregman, J. N., Miller, E. D., \& Irwin, J. A. 2001, ApJ, 553, 125

Canizares, C. R., Fabbiano, G., \& Trinchieri, G. 1987, ApJ, 312, 503

Ciotti, L., Pellegrini, S., Renzini, A., \& D'Ercole, A. 1991, ApJ, 376,380

Cohen, J. G., \& Ryzhov, A. 1997, ApJ, 486, 230

de Vaucouleurs, G., de Vaucouleurs, A., Corwin, Jr. H. G., et al. 1991, Third Reference Catalog of Bright Galaxies (RC3 Catalog)

Fabian, A. C., Hu, E. M., Cowie, L. L., \& Grindlay, J. 1981, ApJ, 248, 47

Fabian, A. C., Stewart, G. C., Nulsen, P. E. J., Itoh, H., \& Canizares, C. R. 1984, Nature, 307, 343

Fabian, A. C. 1994, ARA\&A, 32, 277

Fabian, A. C., Mushotzky, R. F., Nulsen, P. E. J., \& Peterson, J. R. 2001, MNRAS, 321, 20

Feigelson, E. D., Wood, P. A. D., Schreier, E. J., Harris, D. E., \& Reid, M. J. 1987, 312, 101

Finoguenov, A., \& Jones, C. 2000, ApJ, 539, 603

Finoguenov, A., Matsushita, K., Böhringer, H., Ikebe, Y., \& Arnaud, M. 2002, A\&A, 381, 21

Fukazawa, Y., Ohashi, T., Fabian, A. C., et al. 1994, PASJ, 46, L55

Gerhard, O., Kronawitter, A., Saglia, R. P., \& Bender, R. 2001, ApJ, 121, 1936 
Gil'fanov, M. R., Syunyaev, R. A., \& Churazov, E. M. 1987, SvAL, 13, 3

Giraud, E. 1999, ApJL, 524, 15

Ikebe, Y., Ezawa, H., Fukazawa, Y., et al. 1996, Nature, 379, 427

Ikebe, Y., Makishima, K., Fukazawa, Y., et al. 1999, ApJ, 525, 58

Ikebe, Y. 2001, Proc. of Tracing Cosmic Evolution with Clusters of Galaxies, Sesto Pusteria, Bolzano, Italy, July 36, 2001 [astro-ph/0112132]

Kaastra, J. S. 1992, An X-Ray Spectral Code for Optically Thin Plasmas (Internal SRON-Leiden Report, updated version 2.0)

Kaastra, J. S., Ferrigno, C., Tamura, T., et al. 2001, A\&A, 365, L99

Kodama, T., \& Arimoto, N. 1997, 320, 41

Kronawitter, A., Saglia, R. P., Gerhard, O., \& Bender, R. 2000, A\&AS, 144, 53

Lieu, R., Mittaz, J. P. D., Bowyer, S., et al. 1996, ApJL, 458, 5

Liedahl, D. A., Osterheld, A. L., \& Goldstein, W. H. 1995, ApJ, 438, L115

Loewenstein, M., \& Mathews, W. G. 1991, ApJ, 373, 445

Masai, K. 1997, A\&A, 324, 410

Makishima, K., Ezawa, H., Fukazawa, Y., et al. 2001, PASJ, 53,401

Matsumoto, H., Koyama, K., Awaki, H., et al. 1996, PASJ, 48, 201

Matsushita, K., Makishima, K., Rokutanda, E., et al. 1997, ApJ, 488, L125

Matsushita, K., Makishima, K., Ikebe, Y., et al. 1998, ApJ, 499, L13
Matsushita, K., Ohashi, T., \& Makishima, K. 2000, PASJ, 52, 685

Matsushita, K. 2001, ApJ, 547, 693

Matsushita, K., et al. 2002, in preparation (Paper II)

Mewe, R., Gronenschild, E. H. B. M., \& van den Oord, G. H. J. 1985, A\&AS, 62, 197

Mewe, R., Lemen, J. R., \& van den Oord, G. H. J. 1986, A\&AS, 65,511

Nulsen, P. E. J., \& Böhringer, H. 1995, MNRAS, 274, 1093

Raymond, J. C., \& Smith, B. W. 1977, ApJS, 35, 419

Sanders, J. S., \& Fabian, A. C. 2001, MNRAS, in press

Sarazin, C. 1988, X-ray emission from clusters of galaxies (Cambridge University Press)

Smith, R. K., Brickhouse, N. S., Liedahl, D. A., \& Raymond, J. S. 2001, ApJ, 556, 91

Shibata, R. 2000, Ph.D. Thesis, Gakushuin University

Shigeyama, T. 1998, ApJ, 497, 587

Stark, A. A., Gammie, C. F., Wilson, R. W., et al. 1992, ApJS, 79,77

Stewart, G., Canizares, C. R., Fabian, A. C., \& Nulsen, P. E. J. 1984, ApJ, 278, 536

Tamura, T., Kaastra, J. S., Peterson, J. R., et al. 2001, A\&A, 365, L87

Tawara, Y., Akimoto, F., Kumada, A., et al. 1997, in X-ray Imaging and Spectroscopy of Cosmic Hot Plasmas, ed. F. Makino, \& K. Mitsuda (Universal Academy Press, Tokyo), 87

Whitmore, B. C., McElroy, D. B., \& Tonry, J. L. 1985, ApJS, 59,1

Xu, H., Kahn, S., Peterson, J. R., Beher, E., et al. 2001, ApJ, submitted [astro-ph/0110013)] 